\title{
Tomato SIAN11 regulates flavonoid biosynthesis and seed dormancy by interaction with bHLH proteins but not with MYB proteins
}

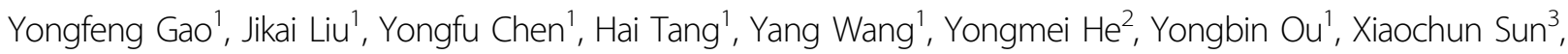
Songhu Wang ${ }^{4}$ and Yinan Yao ${ }^{1}$

\begin{abstract}
The flavonoid compounds are important secondary metabolites with versatile human nutritive benefits and fulfill a multitude of functions during plant growth and development. The abundance of different flavonoid compounds are finely tuned with species-specific pattern by a ternary MBW complex, which consists of a MYB, a bHLH, and a WD40 protein, but the essential role of SIAN11, which is a WD40 protein, is not fully understood in tomato until now. In this study, a tomato WD40 protein named as SIAN11 was characterized as an effective transcription regulator to promote plant anthocyanin and seed proanthocyanidin (PA) contents, with late flavonoid biosynthetic genes activated in 35S::SIAN11 transgenic lines, while the dihydroflavonol flow to the accumulation of flavonols or their glycosylated derivatives was reduced by repressing the expression of SIFLS in this SIAN11-overexpressed lines. The above changes were reversed in 35S::SIAN11-RNAi transgenic lines except remained levels of flavonol compounds and SIFLS expression. Interestingly, our data revealed that SIAN11 gene could affect seed dormancy by regulating the expressions of abscisic acid (ABA) signaling-related genes $S / A B / 3$ and $S I A B I 5$, and the sensitivity to ABA treatment in seed germination is conversely changed by SIAN11-overexpressed or -downregulated lines. Yeast two-hybrid assays demonstrated that SIAN11 interacted with bHLH but not with MYB proteins in the ternary MBW complex, whereas bHLH interacted with MYB in tomato. Our results indicated that low level of anthocyanins in tomato fruits, with low expression of bHLH (SITT8) and MYB (SIANT1 and SIAN2) genes, remain unchanged upon modification of SIAN11 gene alone in the transgenic lines. These results suggest that the tomato WD40 protein SIAN11, coordinating with bHLH and MYB proteins, plays a crucial role in the fine adjustment of the flavonoid biosynthesis and seed dormancy in tomato.
\end{abstract}

\section{Introduction}

The flavonoid compounds, a class of secondary polyphenolic metabolites synthesized via the phenylpropanoid

\footnotetext{
Correspondence: Yinan Yao (yaoya@ms.xjb.ac.cn)

${ }^{1}$ School of Life Science and Engineering, Southwest University of Science and Technology, Mianyang 621010, China

${ }^{2}$ Yunnan Engineering Laboratory for Agro-environment Pollution Control and Eco-remediation, The Innovation Team for Farmland Non-pollution Production of Yunnan Province, Kunming 650201, China
}

Full list of author information is available at the end of the article pathway, fulfill many vital biological functions and mainly include flavonols or their glycosylated derivatives, anthocyanins, and proanthocyanidins (PAs) in the vegetables ${ }^{1}$. Besides producing flower color and providing colors attractive to plant pollinators, flavonoid compounds also play significant roles in ultraviolet-B protection, disease resistance, and plant-microbe interactions, and make possible nutritional and medicinal contribution to human health ${ }^{2}$.

\section{(c) The Author(s) 2018}

(c) (i) Open Access This article is licensed under a Creative Commons Attribution 4.0 International License, which permits use, sharing, adaptation, distribution and reproduction c. in any medium or format, as long as you give appropriate credit to the original author(s) and the source, provide a link to the Creative Commons license, and indicate if changes were made. The images or other third party material in this article are included in the article's Creative Commons license, unless indicated otherwise in a credit line to the material. If material is not included in the article's Creative Commons license and your intended use is not permitted by statutory regulation or exceeds the permitted use, you will need to obtain permission directly from the copyright holder. To view a copy of this license, visit http://creativecommons.org/licenses/by/4.0/. 
The genes encoding the flavonoid biosynthetic enzymes as well as transcriptional factors that regulate the expression of these genes have been identified and characterized from petunia, Arabidopsis, snapdragon, maize, and other plant species ${ }^{2,3}$. It plays a vital role in the spatial and temporal expression of structural genes in flavonoid biosynthesis by forming a ternary MBW complex composed of an R2R3-MYB protein, a protein with basic helix-loop-helix (bHLH) domain and a Trp-Asp (WD)repeat protein. Based on whether be regulated by the MBW protein complex, the genes encoding common enzymes shared by flavonoid biosynthesis pathways can be subdivided into late biosynthetic genes (LBGs; e.g., DFR, LDOX, BAN, TT19, TT12, and AHA10) that are dependent on this complex, and early biosynthetic genes (EBGs; e.g., CHS, $\mathrm{CHI}, \mathrm{F3H}, \mathrm{F3}{ }^{\prime} \mathrm{H}$, and $F L S 1$ ) that are not (Fig. 1$)^{4-6}$.

WD proteins are defined by the presence of four or more highly conserved repeating units usually terminating in a WD dipeptide, and belong to a huge family existed in all eukaryotes ${ }^{7}$. To date, members of this family are involved in many functions like signal transduction, transcription regulation, and cell cycle regulation ${ }^{8}$. The first WD40 gene that functions in regulating anthocyanins biosynthesis was identified in petunia by transposon tagging means and named Anthocyanins11 (AN11), which regulates the pigmentation of the flower ${ }^{9}$. Subsequently, the Transparent Testa Glabra1 (TTG1) locus in Arabidopsis was isolated and was found to encode a WD40 repeat $\operatorname{protein}^{10}$. Previous studies have shown that TTG1 is expressed in all examined tissues of tested plants, and plays various roles in Arabidopsis, including anthocyanin and PA biosynthesis, seed coat mucilage, trichome, and root hair patterning ${ }^{11}$. To date, homologs of TTG1 have been identified and shown species-specific function in different plant species, like ZmPAC1 from Zea mays, MtWD40-1 in Medicago, FaTTG1 in strawberry, and AaTTG1 from Arabis alpine $e^{12-15}$.

As an economically important crop, tomato (Solanum lycopersicum) provide the largest dietary source of lycopene and other carotenoids, like $\beta$-carotene, which serve as an important bioactive compound beneficial to human health ${ }^{16}$. However, in most of the tomato varieties, flavonoids/anthocyanins are generally not present in both tomato fruit peel and flesh tissues ${ }^{17}$. Recently, several members of MBW complex in tomato have been identified and partially characterized as a regulator of anthocyanin biosynthesis. Two paralog genes encoding homologous R2R3-MYB transcription factors (TFs), Anthocyanins1 (SlANT1) and Anthocyanins2 (SlAN2), are both mapped on chromosome 10 of tomato and highly homologous with PhAN2 in Petunia $x$ hybrid ${ }^{18,19}$. Ectopic expression of SIANT1 and SlAN2 can induce anthocyanin biosynthesis in the various organs of the transgenic

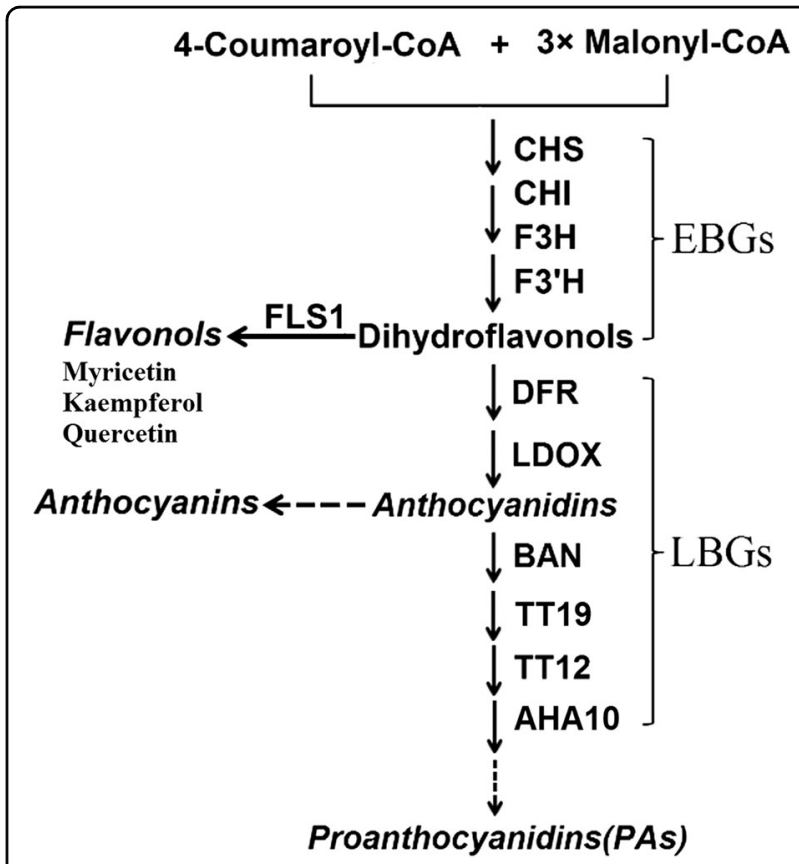

Fig. 1 Simplified flavonoid biosynthetic pathway. First, chalcone synthase (CHS) catalyzes the condensation of one molecule of 4-coumaroyl-CoA with three molecules of malonyl-CoA. The later steps in this pathway are catalyzed by a series of enzymes, leading to the production of three main types of final products: flavonols (including quercetin, kaempferol, and myricetin); anthocyanins; and proanthocyanidins (PAs). CHS chalcone synthase, $\mathrm{CH}$ chalcone isomerase, F3H flavanone 3-hydroxylase, F3'H flavonoid 3'-hydroxylase, DFR dihydroflavanol 4-reductase, FLS flavonol synthase, LDOX leucoanthocyanidin dioxygenase, BAN anthocyanidin reductase, TT19 glutathione-S-transferase, TT12 multidrug and toxic efflux transporter, AHA10 $\mathrm{H}^{+}$ATPase

tomato lines, and SlAN2 mediated the trigger of anthocyanin biosynthesis induced by high light as well as cold in vegetative organs ${ }^{20}$. SlGL3 gene, which encodes a bHLH TF homologous to Arabidopsis GLABRA3 (GL3), could repress anthocyanin accumulation when heterologously expressed in Arabidopsis, suggesting that SIGL3 functions as a repressor of anthocyanins accumulation ${ }^{21}$. More recently, overexpression of another bHLH TF gene wildtype (WT) $A H$ in FMTT271 (a tomato inbred line contains a mutated allele of $A H$, which showed no anthocyanin pigmentation) led to higher anthocyanin accumulation and improved transcript of several anthocyanin biosynthesis genes, demonstrating that $A H$ serves as a key transcriptional regulator of anthocyanin biosynthesis in S. lycopersicum ${ }^{22}$.

Despite much knowledge of the MBW complex model regulating the flavondoid/anthocyanin biosynthesis in many plant species, most of the identified genes belong to the R2R3-MYB and bHLH TF family in tomato until now, little is known about the roles of WD-repeat proteins in regulation of flavonoid/anthocyanin biosynthesis and 
other developmental processes in tomato. In addition, the relative contributions of bHLH, MYB, and WD-repeat protein to the flavonoid/anthocyanin pigment accumulation and its tissue-specific patterns in tomato remain poorly described. In this study, we characterized the molecular function of a tomato WD40 gene, named SlAN11 (for homology to PhAN11) ${ }^{20}$, and demonstrated that it participated in flavonoid biosynthesis in both tomato plant and seeds. In addition, we examined its interactions with bHLH and MYB TFs, and their transcriptional activities by yeast two (Y2H)-/one-hybrid assays. Moreover, our data revealed a novel role of SlAN11 gene in controlling seed dormancy as well as its relation with ABA signaling. Finally, the deficiency of gene transcripts of SlANT1, SlAN2, and SITT8 may shed some light on the absence of anthocyanin accumulation in tomato fruits.

\section{Materials and methods Phylogenetic analysis}

SlAN11 and homologous WD40 proteins were identified from the NCBI Network using the blastp search tool in the reference proteins database. WD-repeat motif sequences of these proteins were defined using the protein prediction program available at the European Bioinformatics Institute (http://www.ebi.ac.uk/interpro/). To analyze the phylogenetic relationship between SlAN11 and WD40 proteins from 16 other species, full-length amino-acid sequences were aligned by MEGA 6 . An unrooted tree was constructed using the neighbor-Joining method, with 1000 bootstrap value.

\section{Material and plant growth conditions}

Tomato (S. lycopersicum) cv Ailsa Craig (LA2838A) was obtained from the TGRC (http://tgrc.ucdavis.edu/). The WT tomato plants and transgenic plants were grown in the greenhouse under long-day conditions $\left(26^{\circ} \mathrm{C}\right.$ day, $18^{\circ}$ $\mathrm{C}$ night; $16 \mathrm{~h}$ light, $8 \mathrm{~h}$ dark), with a relative humidity of $50-60 \%$. Primary transformants $\left(\mathrm{T}_{0}\right)$ and their offsprings were planted under the long-day conditions and then transplanted into the field 4-6 weeks later.

\section{Plasmid construction and tomato transformation}

DNA manipulations were performed by using standard molecular biology techniques ${ }^{23}$. In order to generate the overexpression constructs, SlAN11 (accession No. XM_004235284.3) were amplified from cDNAs by PCR using specific primers (Supplementary Table 1). The 1029 bp fragment obtained by PCR ligated to plant expression vector pBI121 under the transcriptional control of the $35 \mathrm{SCaMV}$ promoter. The resulting construct pBI121$35 S_{\text {Pro: }}:$ SlAN11 was created.

Sequences from SlAN11 cDNA were amplified by PCR for construction of the 35S::SlAN11-RNAi vector. An inverted-repeat target gene fragment was constructed in vector pSKint $^{24}$ and transferred into pBI121 (driven by

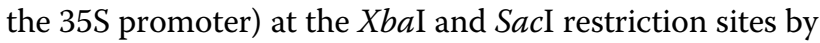
PCR using specific primers (Supplementary Table 1). The resulting construct pBI121-35S Pro: $:$ SlAN11-RNAi was created.

For determining the subcellular localization of SlAN11 protein, the complete open reading frame (ORF) without the stop codon of SlAN11 was amplified by using primers (Supplementary Table 1), incorporating restriction sites $\mathrm{Xba \textrm {I }}$ and $\mathrm{XhoI}$ at the primer ends. The amplified fragments were cloned into the expression vector PTEX-GFP to generate pTEX-SIAN11-GFP.

To isolate SlAN11 promoter from tomato genomic DNA, PCR specific primers were designed (Supplementary Table 1). The location of forward primer was approximately $2 \mathrm{~kb}$ upstream of the translation start site. A 2034 bp SlAN11 promoter was amplified by PCR and ligated to pBI121 plasmid vector and fused with GUS report gene, replacing the $35 \mathrm{SCaMV}$ promoter. Consequently, the GUS expression construct pBI121-SlAN11Pro::GUS was generated.

Above pBI121-35S Pro::SlAN11, pBI121-35S Pro::SlAN11$R N A i$, and pBI121-SlAN11 Pro::GUS plasmids were transferred separately to Agrobacterium tumefaciens EHA105 and the recombinant strains were used to transform tomato according to the method described by Fillatti et $\mathrm{al}^{25}$. The transformed lines with transgene insertion were first selected for kanamycin $(70 \mathrm{mg} / \mathrm{L})$ resistance, and then confirmed by PCR using NPTIIspecific primers.

\section{Quantitative real-time PCR assays}

Quantitative real-time PCR (qRT-PCR) was performed with the IQ SYBR Green Supermix (Bio-rad catalog \#1708882) using the Applied Biosystems Step One RealTime PCR System, with tomato SlUBI3 gene as an internal reference. The relative expression values were determined against the WT sample using the $2^{-\Delta \Delta \mathrm{Ct}}$ method. All the primers used for qRT-PCR analysis are provided in Supplementary Table 2.

\section{$\beta$-glucuronidase staining assay}

Histochemical staining of $\beta$-glucuronidase (GUS) activity of transgenic plants harboring SIAN11 1 Pro::GUS was performed according to described previously ${ }^{26}$. Stained tissues or organs were visualized using a Leica microscope coupled to an insight digital camera.

\section{Subcellular localization of SIAN11}

The plasmid pTEX-GFP as positive control and pTEXSIAN11-GFP were separately transfected into tomato mesophyll protoplasts, essentially as described previously ${ }^{27}$. Protoplasts were tested for SIAN11-GFP 
expression using a confocal microscope at $514 \mathrm{~nm}$ wavelength (LSM 5Exciter, Carl-Zeiss) after being incubated at $23^{\circ} \mathrm{C}$ for $16-18 \mathrm{~h}$.

\section{Anthocyanin assays}

Anthocyanins were extracted from fully expanded leaves and stems of 30-day-old tomato plants and from mature red fruit pericarps separately, and assayed according to the methods described previously ${ }^{28}$. In brief, approximately $200 \mathrm{mg}$ of tissue samples were ground in liquid nitrogen, and anthocyanins were extracted for $48 \mathrm{~h}$ in darkness with shaking in $0.5 \mathrm{~mL}$ of $1 \%(\mathrm{v} / \mathrm{v}) \mathrm{HCl}$ in methanol. A total of $0.4 \mathrm{~mL}$ distilled water and $1 \mathrm{~mL}$ of chloroform was added to the samples to separate the anthocyanins, followed by centrifugation for $5 \mathrm{~min}$ at $3000 \mathrm{rpm}$. The upper aqueous phase was determined by measuring the optical density at A535 nm (A535). Anthocyanin content was calculated using the simple formula (A535)/mg fresh weight.

\section{PA extraction and analysis}

PAs of tomato seeds were determined using by Vanillin$\mathrm{HCl}$ method $^{29}$. A unit of $100-200 \mathrm{mg}$ of ground tomato seeds was extracted with $2 \mathrm{~mL} 60 \%$ ethyl alcohol, sonicated for $30 \mathrm{~min}$. The slurry was centrifuged $(12000 \times g)$ at room temperature, and the $200 \mu \mathrm{L}$ supernatant was transferred to a brown tube which $800 \mu \mathrm{L}$ of methanol: $4 \%$ $\mathrm{HCl}$ solution containing $4 \%$ vanillin was added. After stirring with a tube mixer, and incubated for $30 \mathrm{~min}$ at 25 ${ }^{\circ} \mathrm{C}$, the absorbance at $500 \mathrm{~nm}$ of the red solution was measured by a spectrophotometer. PA content was calculated using the standard curve made by standard PAs (REBIO, R131074).

\section{Flavonoid staining in seedlings}

Seven-day-old seedlings of WT and transgenic tomato seedlings were stained according to previously described method $^{30}$. Seedlings were submerged in aqueous solution containing $0.25 \%$ DPBA $(\mathrm{w} / \mathrm{v})$ and $0.02 \%(\mathrm{v} / \mathrm{v})$ Triton X100 for $5 \mathrm{~min}$, and then washed for $5 \mathrm{~min}$ in distilled water. The roots were visualized using a Confocal Laser Scanning Microscope with an Ar-laser (458/488/514 nm) for green fluorescent protein (GFP) and a $543 \mathrm{~nm} \mathrm{HeNe-}$ laser for yellow fluorescent protein.

\section{Seed germination assays}

The germination assays were performed as described previously $^{31}$. In brief, 100-200 WT and T3 homozygous transgenic seeds of the same maturity were surface sterilized by $75 \%$ ethanol for $1 \mathrm{~min}, 15 \% \mathrm{NaClO}$ for $15 \mathrm{~min}$, and then washed in sterilized water eight times. Seeds were placed on 1/2 Murashige and Skoog medium plates containing the different concentrations of ABA, respectively. Germinated seeds were counted daily for 10 days.

\section{Yeast one-/two-hybrid assays}

Yeast one-hybrid assays were carried out according to the following operation. The full-length CDS (coding sequence) of tomato SlAN11, SlANT1, SlAN2, SlGL3, and SlTT8 were PCR-amplified using primers (Supplementary Table 3). These PCR fragments were cloned into pEG202 vector to obtain LexA DNA-binding domain fusion bait constructs, respectively. The resulting bait construct was transformed into yeast strain EGY48 by LiAc-mediated transformation, and 2-3 days later these yeast strains were tested on selective plate medium or by $\beta$ galactosidase activity assay.

For Y2H assays, the full-length CDS of tomato SlAN11, SlANT1, SlAN2, SlGL3, and SlTT8 were PCR-amplified using primers (Supplementary Table 3). These PCR fragments were cloned into pEG202 (containing DNAbinding domain) or pJG4-5 vector (containing activation domain) to obtain bait or prey constructs. Different combinations of bait and prey constructs were cotransformed into yeast strain EGY48 by LiAc-mediated transformation, and 2-3 days later these yeast strains were tested on selective plate medium or by $\beta$ galactosidase activity assay. Yeast one-/two-hybrid assays and

$\beta$-galactosidase quantitative assays were performed according to the procedures in Current Protocols in Protein Science, 2001. 19.0.1 and the Yeast Protocols Handbook PT3024-1.

\section{Restults}

The SIAN11 gene is a homolog of a regulatory gene involved in flavonoid/anthocyanin synthesis

The full-length SlAN11 gene was about $1436 \mathrm{bp}$, including an ORF of $1029 \mathrm{bp}$, encoding 342 amino acids. Comparison between the genomic DNA and the cDNA demonstrated that SlAN11-lacked introns within the gene sequence like AtTTG1 identified from Arabidopsis and other TTG1 homologs ${ }^{9,10,13,32}$.

At the amino level, the sequence identity between SlAN11 and WD40 protein in other plant species ranged from 77 (AtTTG1) to 99\% (SpTTG1-like), with the highest homologs of solanaceous plants, such as $99 \%$ (Solanum pennellii, SpTTG1-like), 94\% (Nicotiana attenuata, NaTTG1), and $89 \%$ (Petunia $x$ hybrid, PhAN11). Lower sequence identity of TTG1 homologs comes from other plant species, for example: 77\% (Arabidopsis thaliana, AtTTG1); 79\% (Punica granatum, PgWD40); and 80\% (Populus trichocarpa, PtTTG1). Phylogenetic analysis of the amino-acid sequences confirmed that SlAN11 clusters together with the proteins from other plants mentioned above (Fig. 2).

All characterized WD40 protein involved in anthocyanins synthesis have four conserved WD-repeat motifs, like AtTTG1, PhAN11, and MdTTG1 as well as VvWDR1, 


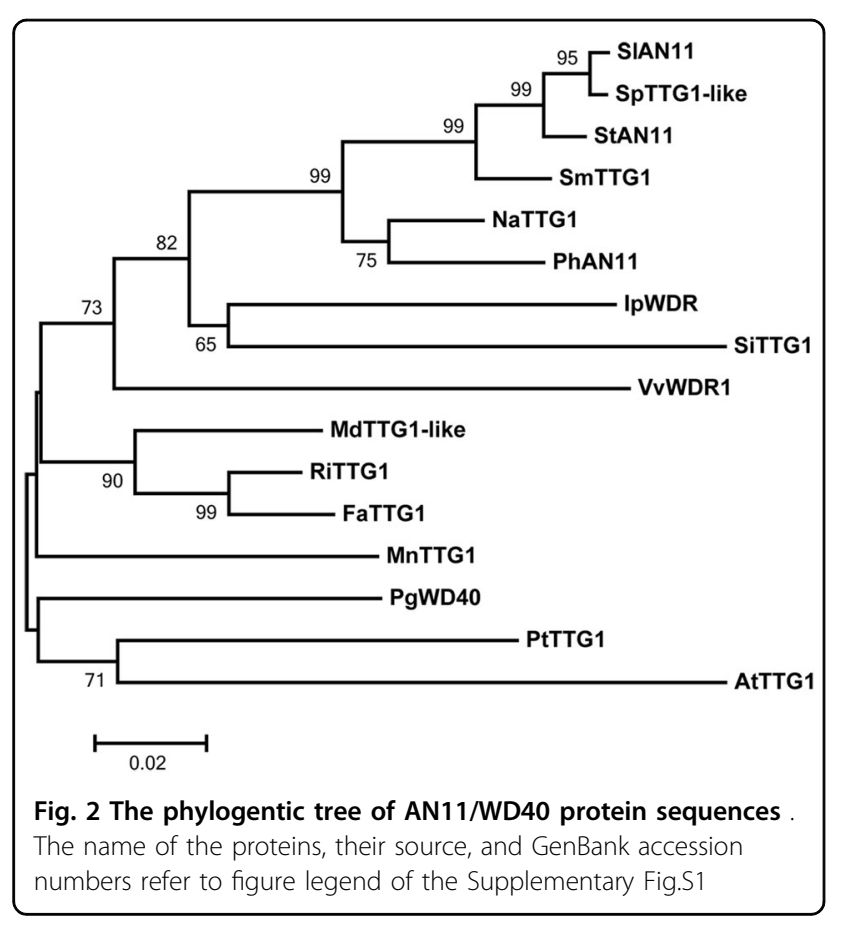

MtWD40-1, PgWD40, etc. ${ }^{9,10,33}$. These four conserved motifs were also found in tomato SlAN11 (Supplementary Figure S1). Moreover, the last two amino-acid residues of these four WD-repeat motif showed high conservation among different species (Supplementary Figure S1), and this was consistent with previous known WD40 proteins related to anthocyanin biosynthesis ${ }^{9,10,32,33}$. The above analysis suggested that the tomato SlAN11 gene is involved in flavonoid/anthocyanin biosynthesis.

\section{SIAN11 is expressed ubiquitously in tomato and SIAN11 protein is targeted to the nucleus}

The transcription of TTG1 is detected in all major organs of Arabidopsis ${ }^{10}$. To study the expression pattern of SlAN11 gene in tomato, we first examined the expression level of SlAN11 in roots, stems, leaves, flowers, seeds, and fruit pericarps at different developmental stages of WT plant by using qRT-PCR. As shown in Fig. 3a, SlAN11 is expressed ubiquitously in all tissues examined, though appears to be highly expressed in leaves, stems, flowers, and seeds.

The expression pattern of SlAN11 gene was further verified by histochemical GUS reporter assay. To do this, the $2034 \mathrm{bp}$ promoter fragment, including the $5^{\prime}$ untranslated region of SlAN11 was cloned and fused to a GUS reporter gene (SlAN11 Pro::GUS). Transgenic tomato plants expressing this construct were generated and the SlAN11 $1_{\text {Pro: }}:$ GUS activity was monitored at different organs. As shown in Fig. 3b-i, GUS staining was detected in all tomato organs, including roots, stems, leaves, flowers (mainly expressed in anther tissue), fruits, and seeds. These results were consistent with the results of qRT-PCR analysis of the tissue expression pattern of SlAN11 gene (Fig. 3a). Previous studies have shown that TTG1 acts as a trichome-promoting factor to control the epidermal cell fate and trichome patterning in Arabidopsis $^{34,35}$. Interestingly, no SlAN11 $1_{\text {Pro }}:$ GUS activity was observed in trichome on the stems, leaves, and sepals (Fig. 3d, f, h), indicating that SlAN11 may not be involved in the control of trichome patterning in tomato.

TTG1 and its homologs have been identified as a core member of TTG1/bHLH/MYB transcriptional complex in Arabidopsis and other plants species ${ }^{5,11}$, which prompted us to determine the subcellular localization of SIAN11 protein. The GFP was fused to the $\mathrm{C}$ terminus of SIAN11 (SlAN11-GFP), and the fusion protein was expressed in tomato mesophyll protoplasts. Compared with the signal of GFP alone, which spread ubiquitously in the protoplasts, the green fluorescent signal of SlAN11-GFP recombinant protein was localized exclusively within the nucleus in the transfected protoplasts (Fig. 4). This observation is consistent with the putative role of SlAN11 that acts as a transcription regulator.

\section{SIAN11 regulates anthocyanin biosynthesis in tomato plants}

To better understand the functions of SlAN11, we generated transgenic tomato plants overexpressing (35S::SlAN11) and downregulating (35S:::SlAN11-RNAi) this tomato gene under the control of the constitutive CaMV 35S promoter. Three independent $\mathrm{T}_{0}$ transgenic lines harboring 35S::SlAN11 (OX-3, OX-4, and OX-5) and 35S::SlAN11-RNAi (Ri-7, Ri-8, and Ri-11) were then randomly selected to generate the $\mathrm{T}_{2}$ homozygous tomato plants for further molecular and phenotypic characterization. Compared with WT plants, SIAN11 overexpression transgenic plants showed enhanced anthocyanin accumulation in leaves and stems, whereas 35S::SlAN11-RNA $i$ transgenic tomato lines severely lacked anthocyanins in the epidermis and in subepidermal layers of leaves and stems (Fig. 5a-c). Moreover, the 5-day-old transgenic seedlings also have shown enhanced anthocyanin accumulation in the cotyledon and hypocotyl (Supplementary Figure S2). Chemical analysis further confirmed these observations. The overexpression of SlAN11 increased anthocyanin accumulation by $50-130 \%$ and $100-160 \%$ in leaves and stems, while anthocyanins content in leaves and stems of 35S::SLAN11-RNA $i$ transgenic lines was decreased by 5- to 8-fold and 23- to 35-fold, respectively (Fig. 5d). Interestingly, although the anthocyanin production in transgenic plants was significantly altered, no change in trichome development was observed (Supplementary Figure S3), as was consistent with no SlAN11 $1_{\text {Pro }}:$ GUS activity in trichome (Fig. 3). To ensure the observed phenotypes correlated 


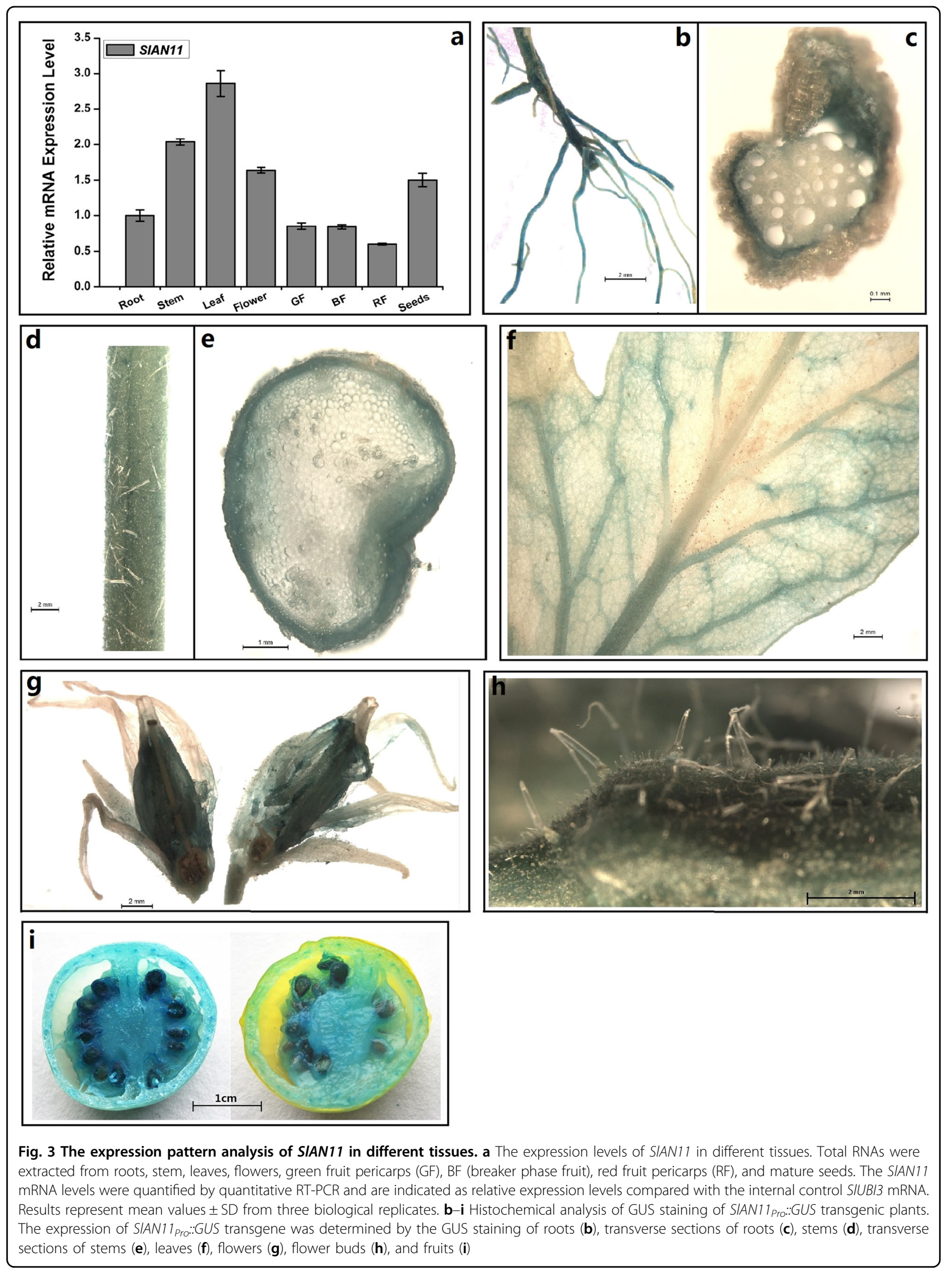




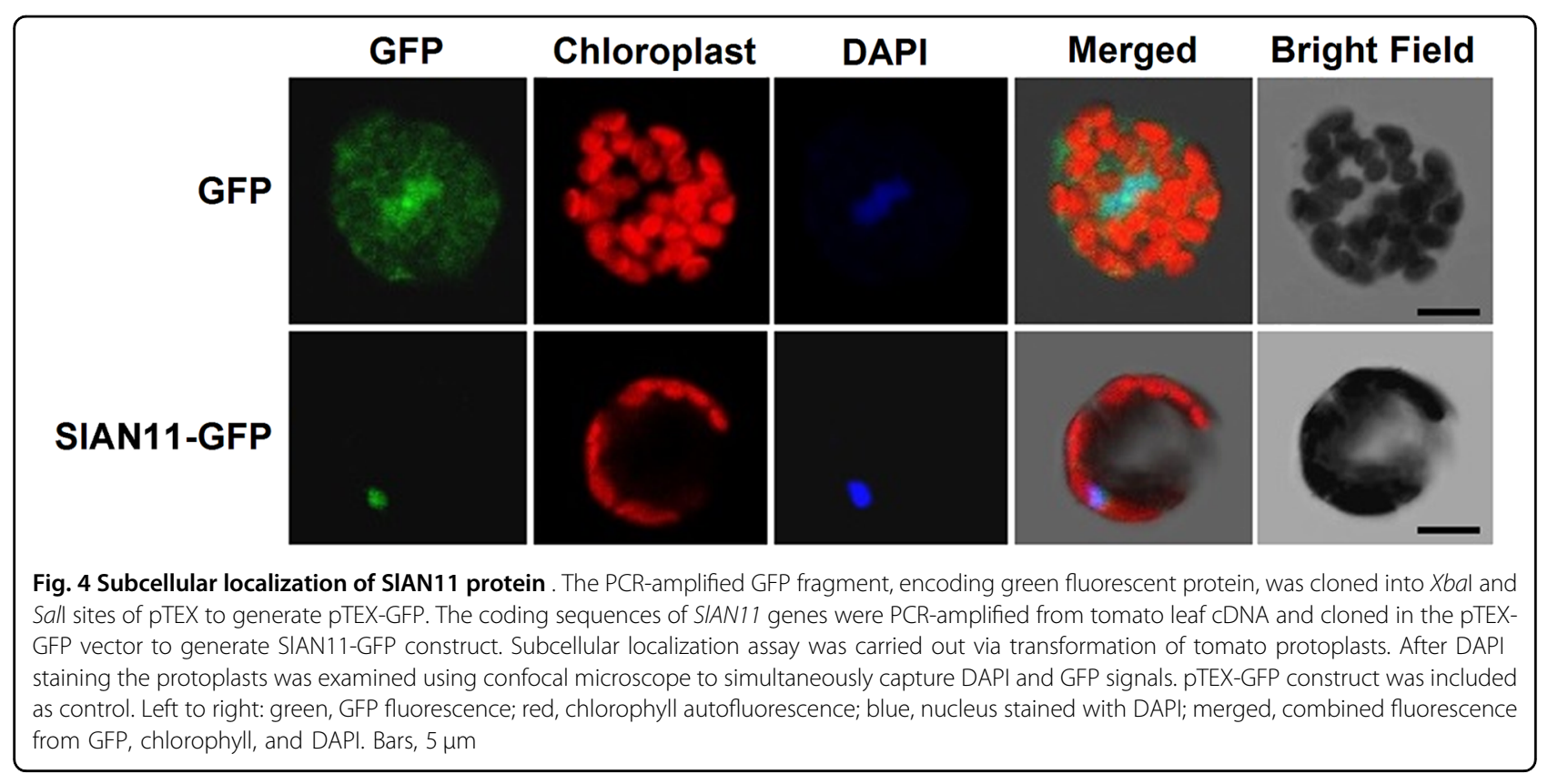

with the upregulated and downregulated transcript, the endogenous SIAN11 mRNA levels in the transgenic plants were determined. Analysis of qRT-PCR revealed a distinct increase and reduction in endogenous SlAN11 transcript levels in 35S::SlAN11 and 35S::SlAN11-RNAi lines compared to that of WT plants, respectively (Fig. 6a).

In Arabidopsis ttg1 mutants, the anthocyanins biosynthetic pathway is blocked at the DFR step, but no effect on transcription of the CHS and CHI genes was observed ${ }^{36}$. We therefore sought to determine whether the overexpression and downregulation of SlAN11 gene could change transcriptional levels of genes involved in flavonoid/anthocyanin biosynthetic pathway (Fig. 1). The transcript abundance of EBGs SlCHS, SlCHI, SlF3'H, and SlFLS was determined by qRT-PCR analysis as well as the LBGs SIDFR. As shown in Fig. 6b-d, expression levels of $S l C H I, S l C H S$, and SlF3'H genes were not significantly altered in 35S::SlAN11 and 35S::SlAN11-RNAi transgenic plants compared with WT. The transcript abundance of key anthocyanin synthesis enzyme, SIDFR, was dramatically increased and decreased in 35S::SlAN11 and 35S:: SlAN11-RNAi transgenic plants, respectively (Fig. 6e). By contrast, the SIFLS transcript was decreased by $50-70 \%$ in 35S::SlAN11 transgenic plants, but not significantly changed in 35S::SlAN11-RNAi transgenic plants (Fig. 6f). As flavonols are synthesized from dihydroflavonols by FLS enzyme, the reduction of SIFLS transcript in 35S::SlAN11 transgenic plants prompted us to analyze changes in flavonol accumulation. Seedlings of WT and transgenic lines were stained with DPBA, a dye used to detect flavonols and their glycosylated derivatives visualized using a laser scanning confocal microscopy ${ }^{30}$. As shown in Fig. 7, compared with WT and 35S::SlAN11-RNAi, the amounts of both K-DPBA (kaempferol) and Q-DPBA (quercetin) fluorescence were significantly decreased in 35S::SlAN11 transgenic seedlings.

\section{SIAN11 regulates PA biosynthesis in seed coats and seed germination}

To further understand the function of SlAN11 in seed coat development, we performed a detailed analysis on seed characters of WT and SlAN11 transgenic lines. As shown in Fig. 8a, compared with WT, overexpression of SlAN11 gene resulted in enhanced PA accumulation in seed coats, while 35::SlAN11-RNAi transgenic seeds exhibited the transparent testa phenotype. Chemical analysis further confirmed these observations. The overexpression of SIAN11 increased PA accumulation by $60-90 \%$ in seeds, while PA content in seeds of $35 S:$ : SlAN11-RNAi transgenic lines was decreased by three- to fivefold (Fig. 8c).

Anthocyanidin reductase encoded by the BANYULS $(B A N)$ gene is the core enzyme in PA biosynthesis ${ }^{37}$. TTG1 can regulate $B A N$ expression in the whole seed coat $^{38,39}$. In addition, Transparent Testa Glabra2 (TTG2) is also required for seed coat PA biosynthesis and acts downstream of TTG1/MYB/bHLH transcriptional complexes $^{34,40}$. We therefore sought to determine whether overexpressing and downregulating SlAN11 gene could affect transcriptional levels of these genes. First, the upregulated and downregulated transcripts of SIAN11 in transgenic tomato seeds were confirmed by qRT-PCR (Fig. 9a). Figure 9b-c showed that the abundance of SIBAN and SITTG2 transcripts dramatically increased in 


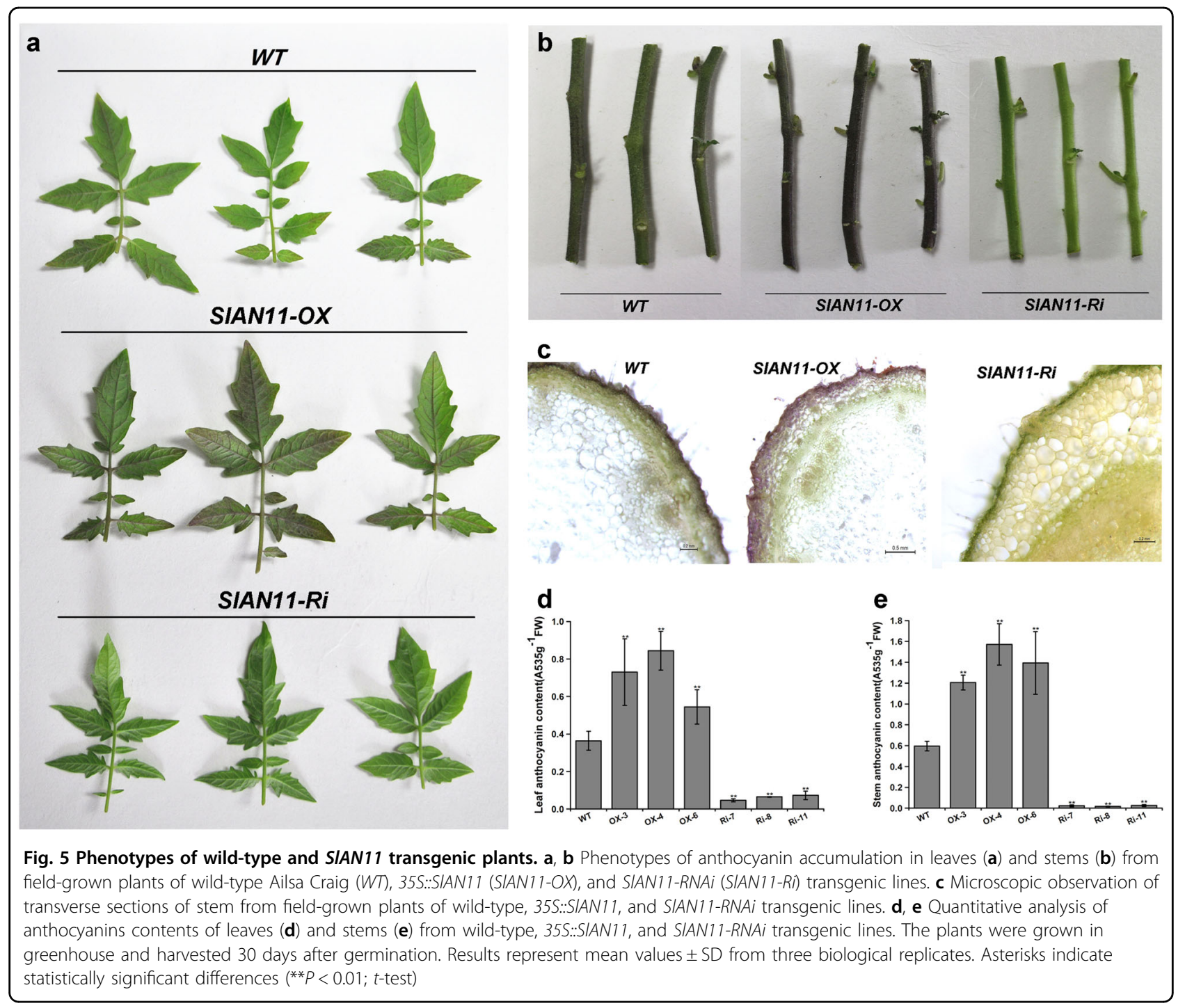

35S::SlAN11 transgenic plants seeds compared with WT. By contrast, their transcript levels were significantly downregulated in 35::SlAN11-RNAi transgenic plants seeds. These results suggest that SlAN11 controls PA biosynthesis in seed coat by regulating its downstream targets genes such as SlBAN and SlTTG2.

In addition, our pilot experiments observed that 35:: SIAN11-RNAi transgenic seeds exhibited higher germination rate than that of the WT and 35S::SlAN11 seeds. To explore the role of SlAN11 in seed germination, WT, 35S:: SlAN11, and 35S:::SlAN11-RNAi seeds were assessed in terms of radicle emergence in absent or presence of various concentrations of ABA. Whether applying ABA or not, 35S::SlAN11-RNAi transgenic seeds exhibited even higher germination rate and less sensitive to exogenous ABA than WT seeds during germination. By contrast, 35S::SlAN11 transgenic plants exhibited a certain degree of germination inhibition, more sensitive to $\mathrm{ABA}$ and reduced in radicle emergence in comparison with WT seeds (Fig. 8b). Upon increase of the exogenous $\mathrm{ABA}$ concentration, the germination of 35S::SlAN11 transgenic seeds was obviously inhibited with a sharper rate than WT seeds, but germination rate of the 35S::SLAN11-RNAi seeds was reduced with a lower rate than WT seeds (Fig. 8b, d, e). In order to further understand the molecular mechanism of SIAN11 in regulating seed germination, we detected the expression levels of SlABI3 and SlABI5 in the seeds of WT and SlAN11 transgenic lines, as ABI3 and ABI5 are two major TFs in ABA signal transduction pathway and involved in ABAdependent growth arrest during germination ${ }^{31,41}$. Compared with WT seeds, the abundance of SIABI3 and SIABI5 transcripts was significantly increased in the 35S::SlAN11, whereas, their transcript levels were dramatically decreased in the seeds of 35S::SlAN11-RNAi transgenic plants (Fig. 9d, e), suggesting that SlABI3 and SlABI5 were positively regulated by SlAN11. 

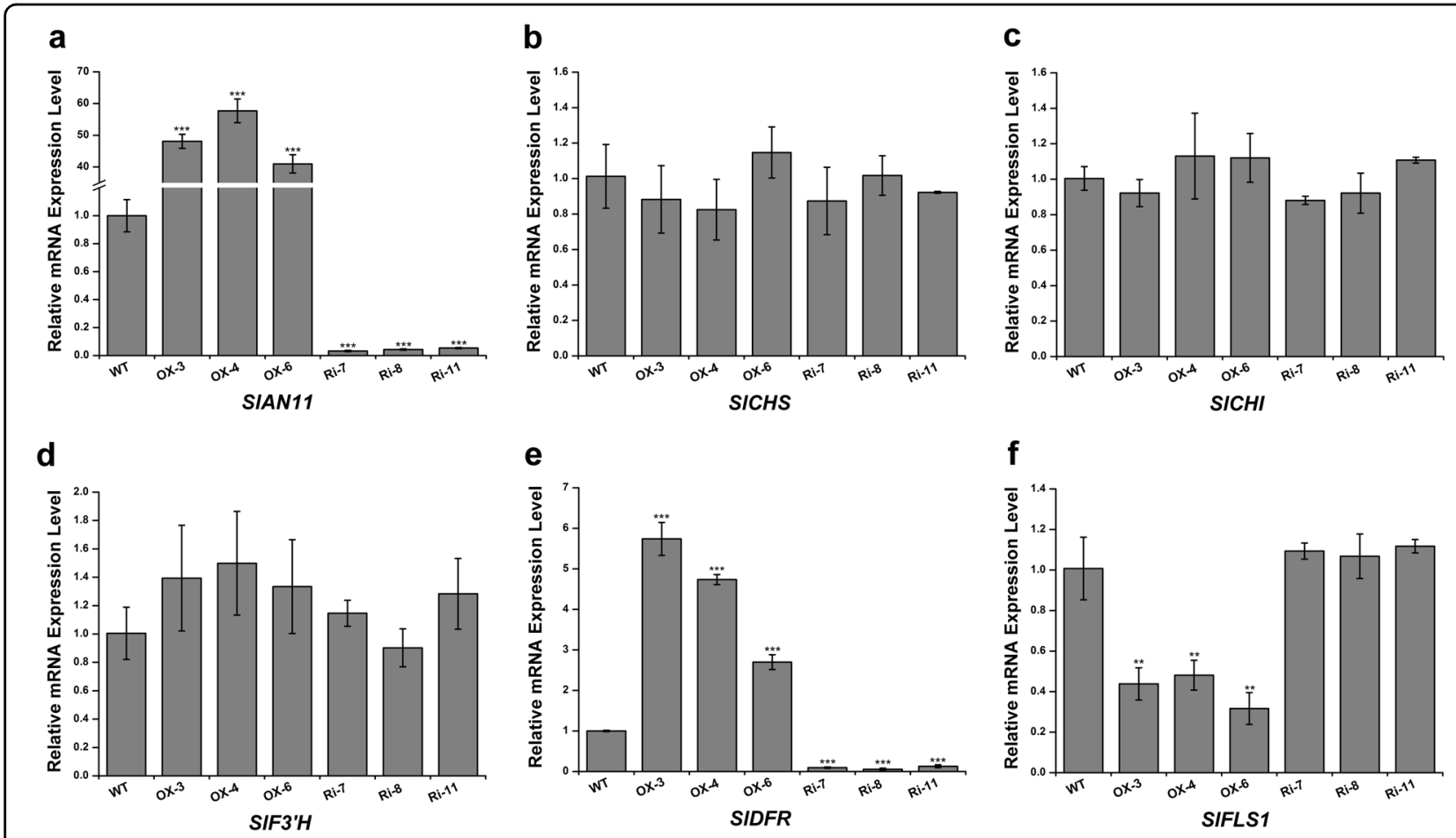

Fig. 6 Expression level analysis of genes involved anthocyanin and flavonol biosynthetic pathway in wild-type and SIAN11 transgenic plants . Total RNA were extracted from seedlings of WT, 35S::SIAN11 (lines OX-3, OX-4, and OX-5), and SIAN11-RNAi (lines Ri-7, Ri-8, and Ri-11) transgenic plants. Quantitative real-time PCR analysis revealed the relative mRNA levels of these genes, including SIAN11 (a), SICHS (b), SICHI (c), SIF3'H (d), SIDFR (e), and SIFLS1 (f). Results represent mean values \pm SD from three biological replicates. Asterisks indicate statistically significant differences $\left({ }^{* * P}<0.01,{ }^{* * *} P<0.001 ; t\right.$-test $)$

SIAN11 physically interacts with bHLH but not with MYB TFs

In Arabidopsis, anthocyanin biosynthesis is modulated by a regulatory complex containing WD40 proteins, MYB and bHLH TFs. We performed $\mathrm{Y} 2 \mathrm{H}$ assays to determine whether SlAN11 can interact with the MYB TFs SlANT1 and SlAN2, as well as the bHLH TFs SITT8 and SIGL3. We first tested the transcriptional activities of these TFs by yeast one-hybrid assay. Yeast colonies expressing SlANT1 and SlAN2 all exhibited strong auto-activation (Fig. 10a), as indicated by the blue color of yeast colonies on the X-Gal plates and growth on selection medium (-Ura-His-Leu). The quantitative analysis on the activity of $\beta$-galactosidase also proved SIANT1 and SlAN2 have strongly transcriptional potential. By contrast, SIAN11 and bHLH TFs SITT8 and SIGL3 proteins did not show auto-activation (Fig. 10a).

Next, the $\mathrm{Y} 2 \mathrm{H}$ system was constructed to assess the interaction between SIAN11 and the MYB TFs SlANT1 and SIAN2, as well as the bHLH TFs SITT8 and SIGL3. The result showed that SIAN11 interacted with all of the bHLH TFs tested, but not with the MYB TFs SIANT1 and SlAN2 (Fig. 10b). The $\beta$-galactosidase activity also indicated that SIAN11 and the bHLH TFs SITT8 and SIGL3 have strongly interactions but not MYB TFs.
In addition, we also tested whether the bHLH TFs SITT8 and SIGL3 interacts with the MYB TFs SIANT1 and SIAN2. The results indicated that the MYB TFs SlANT1 and SlAN2 interacted with all of the bHLH TFs tested (Fig. 10c). Interestingly, bHLH proteins formed homodimers as well as heterodimers with SITT8 or SIGL3. The interactions were also confirmed by quantification of $\beta$-galactosidase activity measurements. Taken together, our results suggest that the SlAN11 protein specifically interacts with bHLH but not with MYB TFs. Moreover, the bHLH TFs interacts with both SIAN11 and the MYB TFs, and can also homodimerize or heterodimerize with related bHLH proteins.

\section{Modification of SIAN11 alone does not affect anthocyanin biosynthesis in tomato fruits}

The altered flavonoid compound accumulation in the leaves, stems, and seeds of SlAN11 transgenic plants prompted us to investigate the effect of SlAN11 on anthocyanin accumulation in tomato fruits. However, unlike the results with the vegetative tissues and seeds, overexpressing and downregulating SlAN11 gene did not influence anthocyanin contents in tomato fruits (Fig. 11a, Supplementary Figure S4). Chemical analysis further 


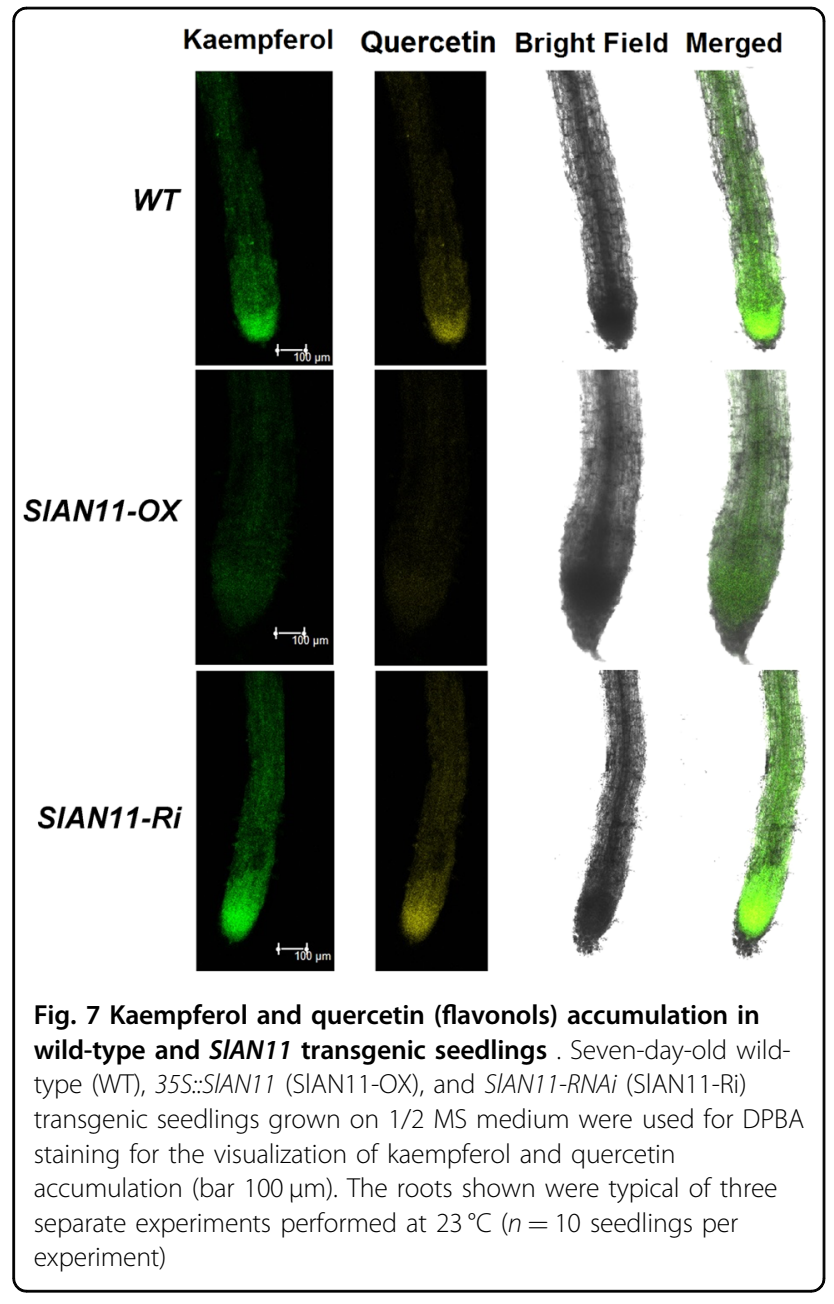

confirmed these observations. Anthocyanin accumulation in fruits was not significantly altered in 35S::SlAN11 and 35S::SlAN11-RNAi transgenic plants compared with WT (Fig. 11b). These results indicated that there exist other factors that function primarily in fruit tissues. In order to further determine the roles of the components of the MBW complex in anthocyanin production in tomato fruits, the expression levels of genes encoding WD40 protein (SlAN11), MYB TFs (SlANT1 and SlAN2), and bHLH TFs (SlTT8 and SlGL3) in different tissues of WT tomato plants was analyzed by using the qRT-PCR. As shown in Fig. 11c, unlike SlAN11 and SlGL3 genes, which were expressed in all tissues tested, the expression patterns of SlANT1, SlAN2, and SITT8 genes showed significant differences among tomato tissues. The transcripts of SlANT1, SlAN2, and SITT8 genes were mainly confined to roots (except SlTT8), stems, leaves, and flowers, while almost no expression was detected in pericarps of green fruits (except SlAN2), break fruits, red fruits, and seeds. Since anthocyanins normally do not accumulate in tomato fruits, our results suggested that the MYB TFs (SIANT1 and SIAN20, and the bHLH TF (SITT8) may be important factors that affected the anthocyanin biosynthesis in tomato fruits.

\section{Discussion}

SIAN11 may modify flavonoid compound levels largely through the regulation of late genes in flavonoid biosynthesis

Anthocyanin biosynthesis is transcriptionally governed by the ternary MBW protein complex, which is constituted by one R2R3-MYB protein like PRODUCTION OF ANTHOCYANIN PIGMENTS $1 / 2$ (PAP1/2), or MYB113/114, one bHLH protein like TT8, GLABROUS 3 (GL3), and one WD protein like TTG1. To date, some genes encoding WD40 protein have been characterized in different plant species, such as Arabidopsis ${ }^{10}$, apple ${ }^{33}$, strawberry ${ }^{14}$, and Chinese bayberry ${ }^{42}$. The above researches all suggest that WD40/TTG1 may play an important role in the biosynthesis of anthocyanins/PAs in plant. In our study, the SlAN11 gene encoding a WD40 repeat protein in S. lycopersicum was identified with the function of regulating anthocyanin/PA biosynthesis by reverse genetics approach.

In Arabidopsis, the expression level of TTG1 is indistinguishable throughout the plant ${ }^{10}$. In tomato, SlAN11 is expressed either in the tissues of rich anthocyanins (leaf, stem, and seed) or in the tissues of less abundant anthocyanins (root, flower, and fruit), and its expression level is similar in all tissues, though highly expressed in leaves, stems, flowers, and seeds (Fig. 3a). These results were consistent with the results of histochemical GUS reporter assay (Fig. 3b-i). Under normal conditions, tomato mainly accumulates anthocyanins/PAs in stems, leaves, and seeds. So, its expression pattern is consistent with anthocyanin accumulation in these tissues, suggesting that SlAN11 is involved in anthocyanin biosynthesis in tomato.

The Arabidopsis ttg1 mutant lacks anthocyanins/PAs in all tissues, including the stems, leaves, and seeds ${ }^{36,43}$. The SIAN11-RNAi transgenic lines severely lack anthocyanins/ PAs in leaves, stems, and seeds (Figs. 5 and 8, Supplementary Figure S2). In contrast, overexpression of SlAN11 in WT tomato seedlings resulted in more anthocyanin accumulation in leaves and stems but not in tomato fruit than WT plants (Figs. 5 and 11b). In Arabidopsis ttg1 mutant, whose anthocyanin biosynthesis is blocked at the DFR step, the transcript of upstream genes showed unaffected, like $C H S, C H I, F 3^{\prime} H$, and $F L S 1^{36}$. Similarly, the LBG SIDFR was remarkably downregulated in SlAN11$R N A i$ transgenic lines, and upregulated in 35S::SlAN11 transgenic lines while the transcription levels of EBGs $\mathrm{CHS}, \mathrm{CHI}$, and $\mathrm{F} 3^{\prime} \mathrm{H}$ were unaffected in these transgenic lines (Fig. 6b-e). However, the transcript of SlFLS1, one EBG gene, was not affected by MBW complex in other model plant species, was remarkably decreased in 


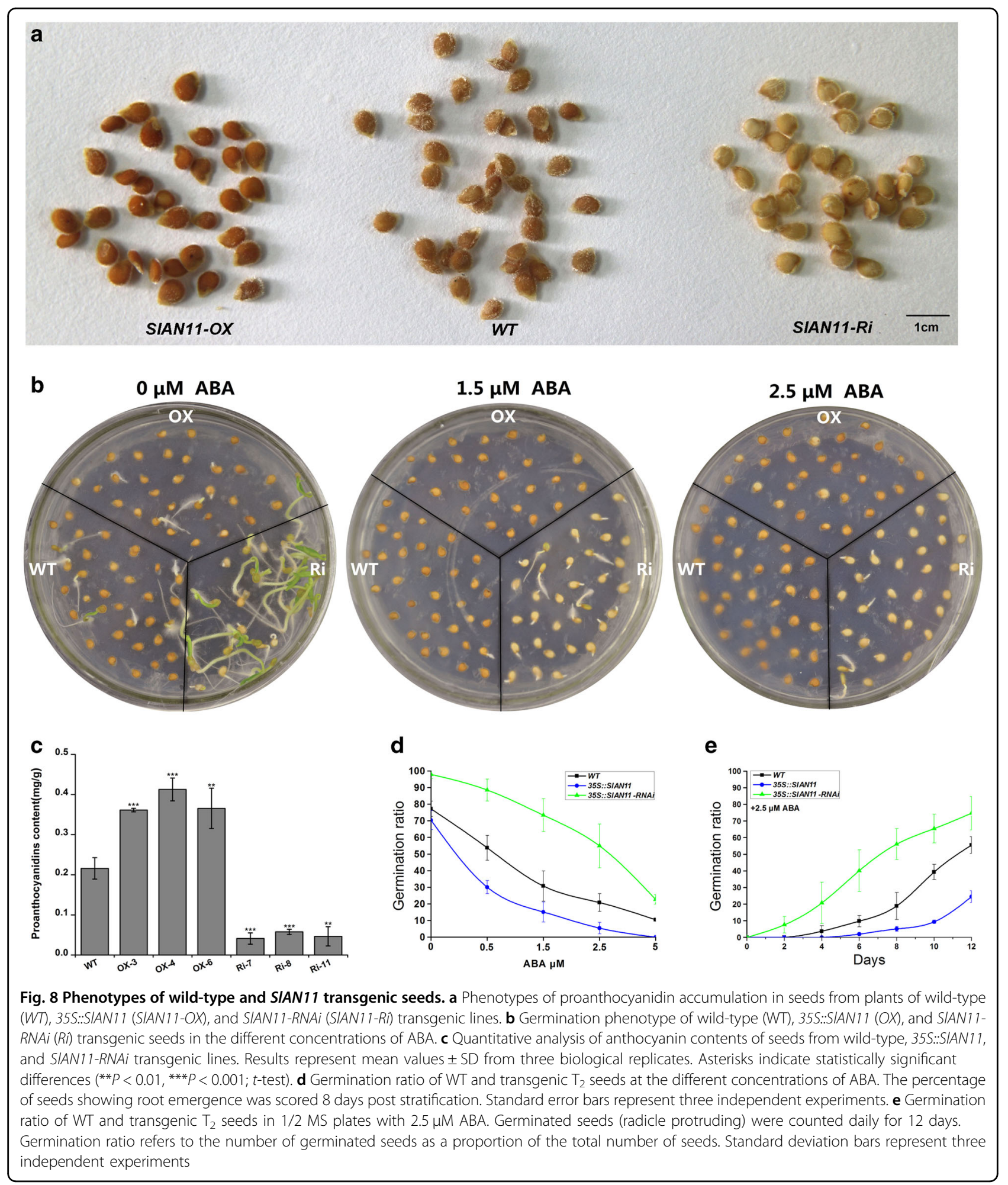

35S::SlAN11 transgenic plants, but not significantly changed in SlAN11-RNAi transgenic plants (Fig. 6f). Accordingly, flavonol staining analysis also revealed that the kaempferol and quercetin concentration all were significantly decreased in 35S::SlAN11 plants in comparison with the WT and SlAN11-RNAi transgenic plants (Fig. 7). These findings suggest that the high anthocyanin content in 35S::SlAN11 transgenic plants may result from modification of dihydroflavonol flow, increased flow to anthocyanins by improved SIDFR 


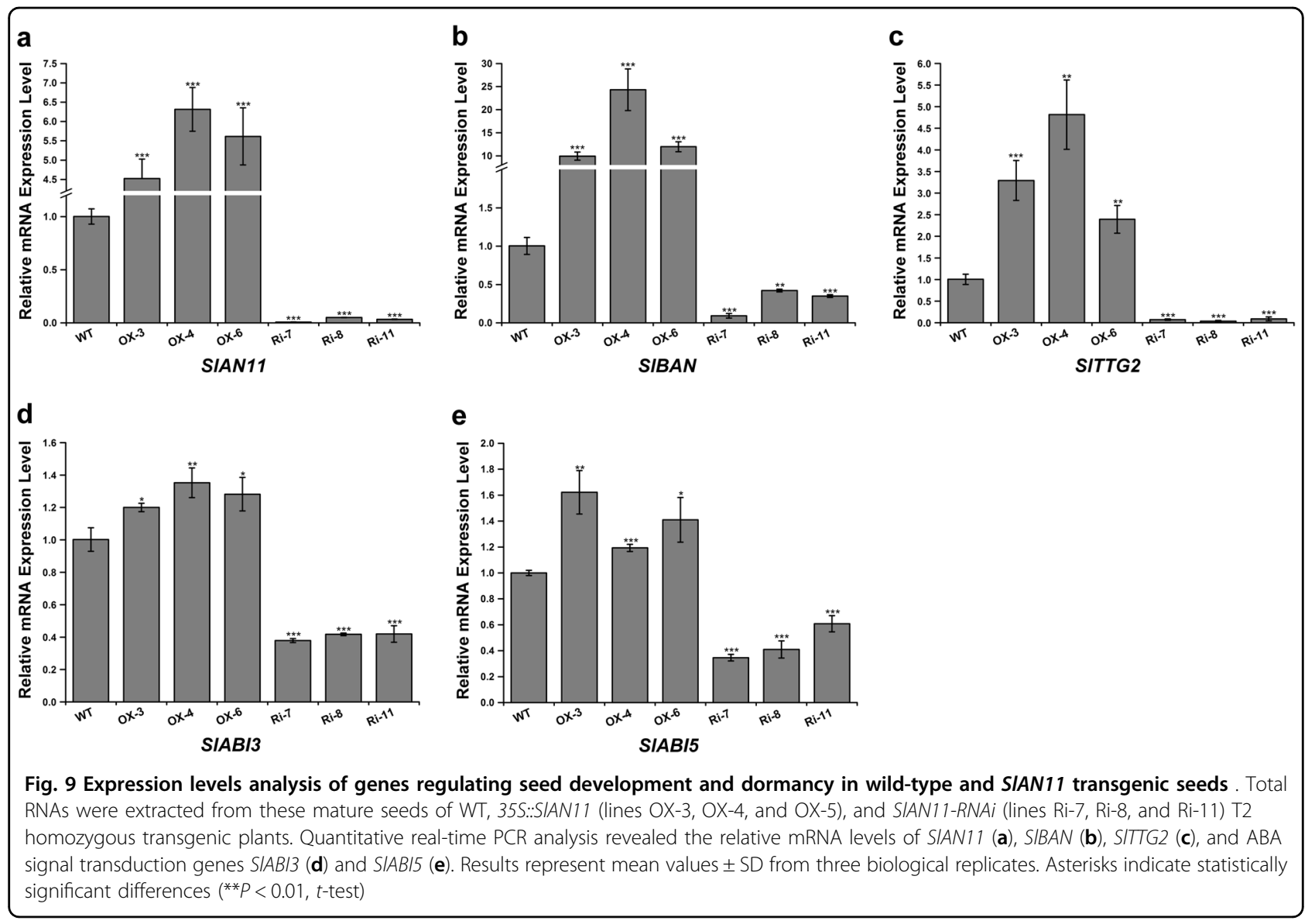

transcript but reduced flow to the accumulation of flavonol or its glycosylated derivatives by repressing the expression of SIFLS.

The accumulation of PA compounds in seeds is also regulated by $\mathrm{MBW}$ protein complexes ${ }^{11,38}$. In addition, downstream direct targets of MBW protein complexes, including other TFs such as the TTG2 WRKY protein, positively regulate the accumulation of $\mathrm{PAs}^{34,40}$. In particular, our results showed that the transcript abundance of the PA-specific gene $B A N$ and the regulatory gene SITTG2 were strongly reduced in 35S::SlAN11-RNAi transgenic seeds, which finally led to a deficiency of PAs in seeds (Fig. 8a, c). In contrast, their transcript levels were dramatically increased in 35S::SlAN11 transgenic seeds, which resulted in more PA accumulation in seed coats (Fig. 9b, c). Taken together, our data suggest a key role for SlAN11 in the governing of seeds' PA biosynthesis, with regulating directly its downstream targets genes SIBAN and SITTG2.

In addition, TTG1 can also regulate TT8 expression in Arabidopsis by taking part in the MYB-bHLH complexes interacting with the TT8 promoter, and the activity of the TT8 promoter is severely affected in $\operatorname{ttg} 1$ mutant $^{44,45}$. In our study, the abundance of SlTT8 transcript significantly increased in the 35S::SlAN11 transgenic plants, and dramatically decreased in 35S::SlAN11-RNAi transgenic plants (Supplementary Figure S5c), suggesting that SlTT8 was positively regulated by SIAN11. However, the transcription levels of another bHLH gene SlGL3 and MYB genes SlANT1 and SlAN2 were not significantly changed in these transgenic lines (Supplementary Figure S5). These findings suggest that SIAN11 influence anthocyanin biosynthesis in tomato not only by forming a MBW complex with SITT8 but also by regulating the transcription of SITT8 gene.

\section{The function of SIAN11 in trichome formation}

WD40 repeat proteins in Arabidopsis play a key role in trichome formation, but this function was not observed in some other plant species $^{46}$. Arabidopsis ttg1 mutant exhibited hairless phenotype in leaves and stems ${ }^{10}$. Besides glandular trichomes similar to Arabidopsis plants, tomato has non-glandular unicellular trichomes on stem and leaves ${ }^{47,48}$. Our study indicated that overexpression or RNAi of SlAN11 gene didn't affect trichome distribution on young leaves and stems (Supplementary Fig-

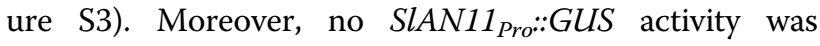
observed in trichome on the stems, leaves, and sepals 


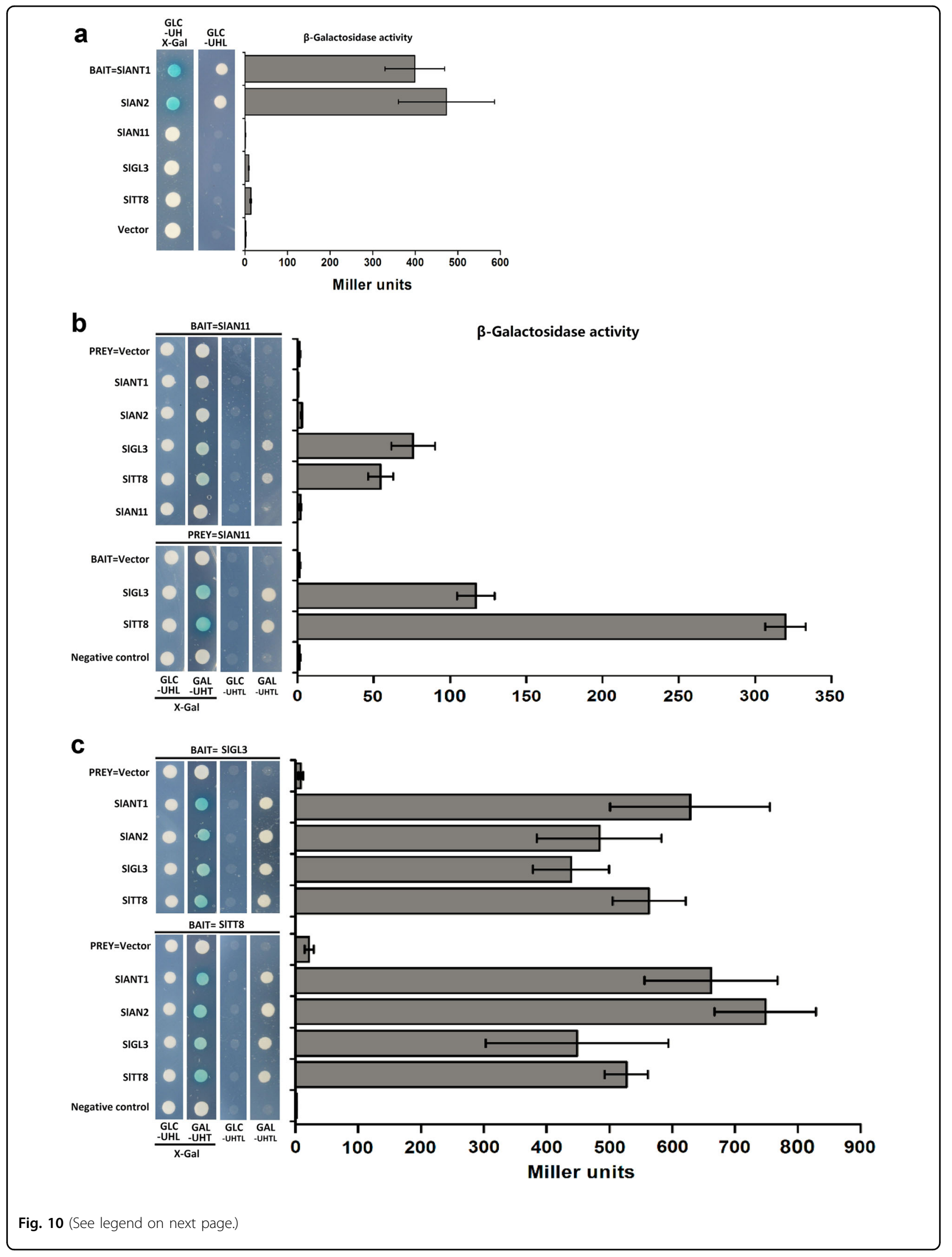


(see figure on previous page)

Fig. 10 The interaction of SIAN11 with the bHLH TFs but not with the MYB TFs in yeast. a Transcriptional activity of SIAN11, SIANT1, SIAN2, SIGL3, and SITT8 determined by the ONPG assay in yeast. Yeast strain EGY48 containing the pSH18-34 reporter plasmid was transformed pEG202SIAN11, pEG202-SIANT1, pEG202-SIAN2, pEG202-SIGL3, and pEG202-SITT8, respectively. Yeast colony grown on X-Gal plates were showed on the left panel with blue color indicating activation of LacZa marker gene by SIANT1 or SIAN2. b SIAN11 has interaction with SIGL3 and SITT8 but not with SIANT1 and SIAN2. c bHLH TFs SIGL3 and SITT8 interact with the MYB TFs SIANT1 and SIAN2, and can also homodimerize or heterodimerize with SIGL3 or SITT8. The interaction was determined by the oNPG assay. Results represent mean values \pm SD from three independent a-galactosidase assays

a

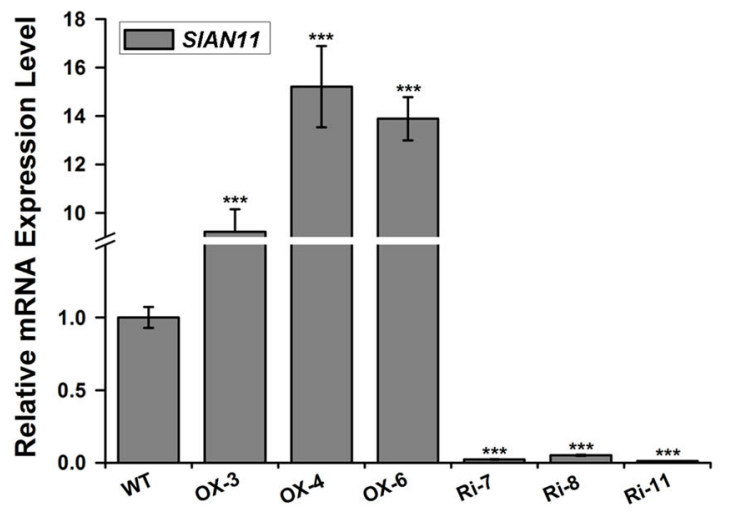

b

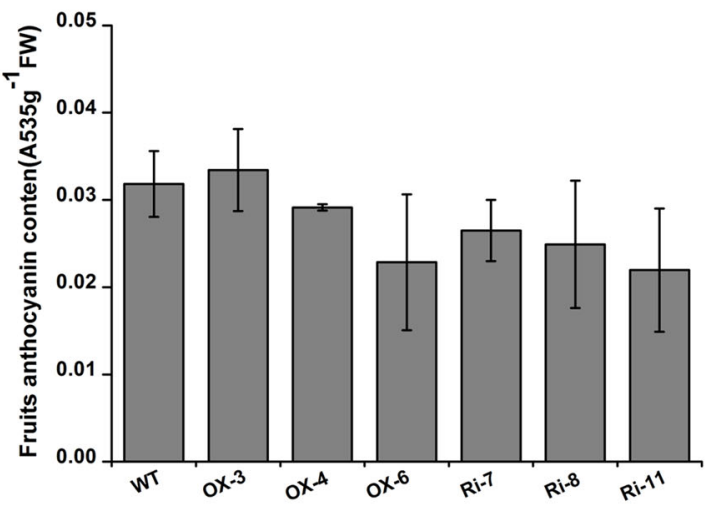

C

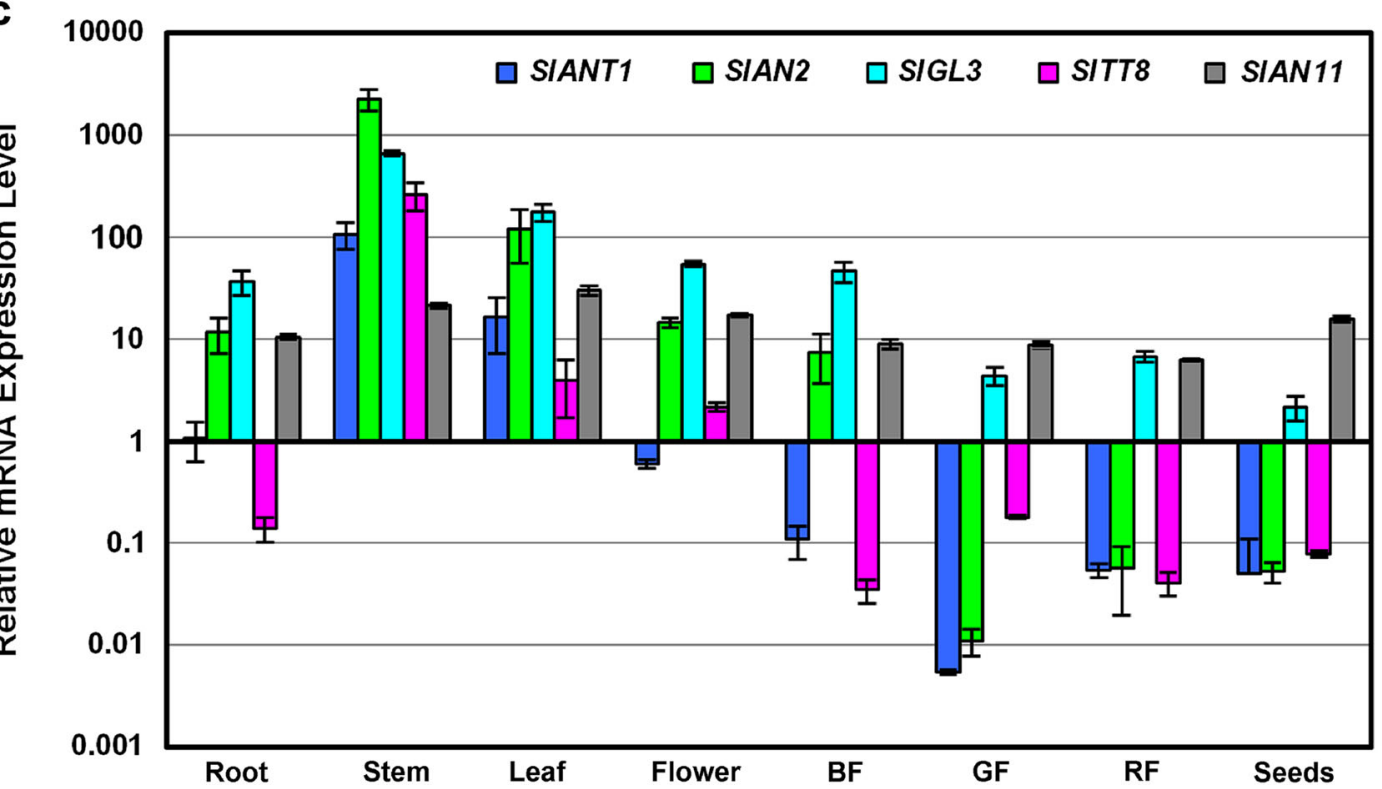

Fig. 11 SIAN11 could not enhance anthocyanins a ccumulation in tomato fruits. a Expression level analysis of SIAN11 in wild-type and SIAN11 transgenic fruits. Total RNAs were extracted from these red fruit pericarps of WT, 35S::SIAN11 (lines OX-3, OX-4, and OX-5), and SIAN11-RNAi (lines Ri-7, Ri-8, and Ri-11) T2 homozygous transgenic plants. b Quantitative analysis of anthocyanin contents of red fruit pericarps from wild-type, 35S::SIAN11, and SIAN11-RNAi transgenic lines. c The expression pattern analysis of SIAN11, SIANT1, SIAN2, SITT8, and SIGL3 in different tissues. Total RNAs were extracted from roots, stem, leaves, flowers, green fruit pericarps (GF), BF (breaker phase fruit), red fruit pericarps (RF), and mature seeds. Relevant mRNA levels were quantified by quantitative RT-PCR and are indicated as relative expression levels compared with the internal control S/UB/3 mRNA. The vertical axis is log scale. Results represent mean values \pm SD from three biological replicates. Asterisks indicate statistically significant differences *** $P<0.001 ;$ t-test) 


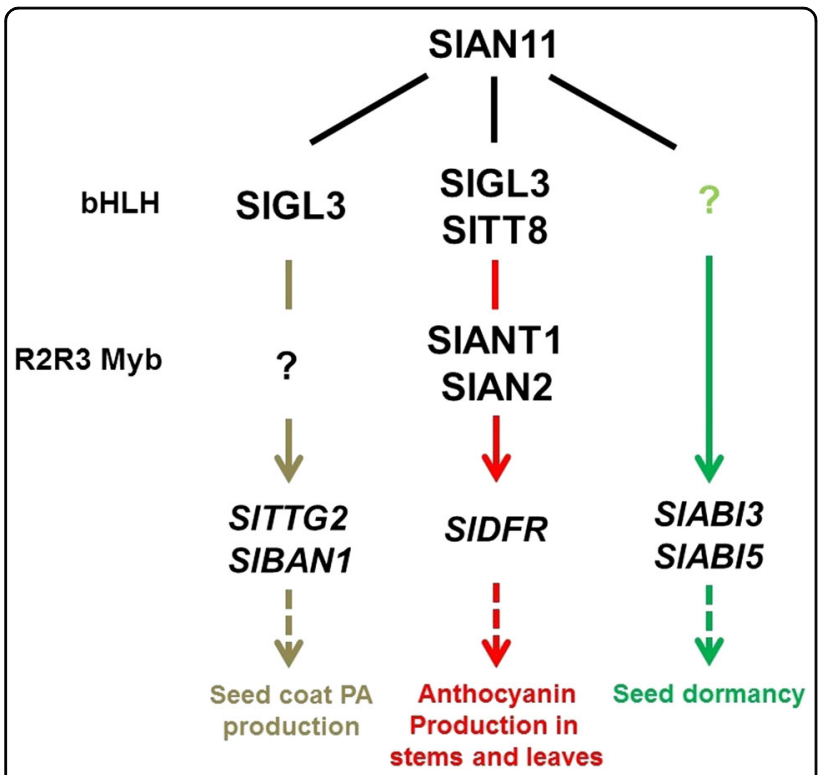

Fig. 12 The model for SIAN11-dependent regulation of anthocyanin/PA accumulation and seed dormancy. Solid lines indicate interactions between members of a complex. Solid arrows indicate positive regulations. Dashed arrows indicate a multi-step differentiation pathway. Colored lines and arrows indicate specific regulator combinations and the pathway controlled

(Fig. 3d, f, h). Our result was consistent with previous studies on Medicago truncatula and petunia. MtWD40-1 mutations in $M$. truncatula didn't pose impacts on the trichome development and distribution in leaves and petioles $^{13}$. Modification of gene expression of AN11, a WD40 protein, also caused little effects in trichome phenotype in petunia 9 .

Like AN11 in petunia and PAC1 in maize, SlAN11 is a single-copy gene, because no other SlAN11 gene loci were recovered in $S$. lycopersicum genome databases by BLASTN analysis with the SlAN11 nucleotide sequence as query. In addition, two new WD40 repeat proteins with more than $60 \%$ identity were recovered when BLASTP query was conducted using deduced amino-acid sequence of SlAN11. SlAN11-like protein (GenBank accession: XP_004235027) was $77 \%$ identical to SlAN11 at the amino-acid level, and another WD40 protein LWD1 (GenBank accession: XP_004238433) showed 61\% identity to SIAN11. Therefore, it is possible that the lack of trichome change in the SlAN11 overexpression and RNAi transgenic lines is due to genetic redundancy.

\section{SIAN11 regulates seed dormancy and cross talk with ABA signaling}

During embryogenesis, TTG1 not only affects biosynthesis of PAs but also affects seed dormancy. In Arabidopsis, the ttg1 mutant reduced seed dormancy, as ascertained by a lower requirement for after-ripening and a higher germination rate ${ }^{49}$. In our study, 35:: SlAN11-RNAi transgenic seeds with transparent testa exhibited reduced dormancy and higher germination rate than WT seeds, whereas 35S::SlAN11 transgenic seeds with more PA accumulation in seed coat exhibited a certain degree of germination inhibition (Fig. 8). Some studies have shown that seed coat pigmentation was positively correlated with seed dormancy in crop plants. For example, red seeds of charlock (Sinapis arvensis L.) exhibit a reduced dormancy compared with black seeds $^{50}$. In legumes, white seeds imbibe more rapidly than colored seeds and then germinate earlier. White seeds also suffer greater imbibition damage, as measured by higher solute leakage, which affects their vigor and viability ${ }^{51,52}$. In Arabidopsis, the reduced dormancy of testa mutants (such as $t t$ mutants and $t \operatorname{tg} 1$ ) is closely related to increasing water penetration and a reduced thickness of the testa ${ }^{49}$. In legumes, water-impermeable seed dormancy was extensively studied and has been attributed to the presence of flavonoid compounds in the seed coat ${ }^{52}$. In tomato, the absence of condensed tannins in the three anthocyaninsless mutant (ah, aw, and $b l s$ ) seeds contribute to increased water permeability and the rapid water uptake and germination ${ }^{53}$. Therefore, it is possible that the permeability and thickness of the testa are affected by the flavonoid compounds (PAs) and structural elements altered in the mutants, which may lead to effects on germination.

The phytohormone ABA plays a vital role in promoting seed dormancy and inhibiting seed germination $^{54}$. 35::SlAN11-RNAi transgenic seeds exhibited less sensitive to exogenous ABA and germinated earlier than WT seeds, in contrast, much more severe inhibition on the ABA-dependent germination was observed in the 35S::SlAN11 transgenic seeds. These results indicated that SlAN11 is a key factor that can both control PA contents in seed coats and regulate seed dormancy and germination in an ABA-dependent way. In ABA signal transduction pathway, Abscisic Acid Insensitive3 (ABI3) and ABI5 are two major TFs and execute ABAdependent growth arrest during germination ${ }^{31,41}$. The abi3/vp1 and abi5 mutant seeds are desiccation-intolerant, insensitive to ABA, and usually germinate precociously in Arabidopsis and maize ${ }^{54,55}$. In addition, Chen's data suggested that abundance of $A B I 3$ transcript was upregulated in developing seeds of Arabidopsis ttg1 mutant ${ }^{56}$. Interestingly, the abundance of SlABI3 and SlABI5 significantly dramatically decreased in SlAN11-RNAi plants compared with WT seeds, whereas, their transcript levels were increased in the 35S::SlAN11 transgenic plants (Fig. 9d, e). These results suggested that SIABI3 and SlABI5 were positively regulated by the SlAN11. In Arabidopsis, a study indicated that PAs could inhibit seed germination by 
sustaining a high level of ABA contents ${ }^{57}$. Taken together, our results provided molecular evidences to illuminate how SIAN11 regulates seed dormancy in tomato through ABA signaling pathway.

\section{SIAN11-dependent MBW complex is required for anthocyanin accumulation in tomato fruits}

WD40 is a crucial component of the MBM protein complex. In Arabidopsis, TTG1 protein interacts with both the bHLH TFs (TT8 and GL3) and the MYB TF (TT2) to regulate the expression of anthocyanin and PA biosynthetic genes ${ }^{45}$. However, in most other plants studied, the WD40 protein only interacts with the bHLH TFs and not with the MYB $\mathrm{TFs}^{33,58,59}$. $\mathrm{Y} 2 \mathrm{H}$ assays proved that SIAN11 interacts only with bHLH TFs in tomato, including SITT8 and SlGL3 (Fig. 10b). Interestingly, bHLH TFs, including SITT8 and SIGL3 were found to interact with SIAN11, as well as with MYB TFs SIANT1 and SIAN2, and could also homodimerize or heterodimerize with SITT8 or SlGL3 protein (Fig. 10c). These results were similar to those of other recent observations and studies ${ }^{33,60}$.

Recent research indicated high positive correlation between the anthocyanin accumulation and the expression level of WD40 in pomegranate fruit ${ }^{3}$. However, no remarkable alterations in anthocyanin contents were discovered in $35 S::$ SlAN11 or 35S::SlAN11-RNAi transgenic tomato fruits (Fig. 11b, Supplementary Figure S4). The result leads to the conclusion that SlAN11 alone could not enhance anthocyanin biosynthesis in tomato fruits. So what caused the absence of anthocyanin accumulation in tomato fruits? It is known that a complex containing a MYB protein, bHLH, and WD40 TF (MBW complex) is necessary for the appropriate regulation of the biosynthesis of anthocyanins in various plant species. Therefore, we examined the expression patterns of SlAN11-, SlANT1-, and SlAN2-encoded MYB TF, as well as SlTT8- and SlGL3-encoded bHLH TF in different tissues of WT tomato. Interestingly, SlANT1, SlAN2, and SITT8 transcripts were mainly confined to root (except SlTT8), stems, leaves, and flowers, with almost no expression in green fruit (except SlAN2), break fruits, red fruits, and seeds (Fig. 11c). By contrast, like SlAN11, SlGL3 was expressed ubiquitously in all organs examined. Our results implied that the MYB TFs (SIANT1 and SlAN2) and the other bHLH TF (SITT8) may be important factors that affected the anthocyanin biosynthesis in tomato fruits. In addition, some studies have also shown that the overexpression of SIANT1 or AtMYB75/PAP1 in tomato resulted in high levels of anthocyanins in their vegetative tissues, but limited purple coloring on pericarp of tomato fruits ${ }^{18,61}$. Overexpression of the SlAN2 in tomato led to the same phenotype in vegetative tissues as SlANT1, but unexpectedly, no anthocyanin accumulation was noted in tomato fruits ${ }^{62}$. Heterologous expression of BoPAP1 in tomato induced stamen-specific anthocyanin accumulation, and observed the consistency of SlTT8 expression and anthocyanin accumulation in purple stamens $^{63}$. Together, these results lead to the conclusion that low expression of SIANT1, SlAN2, and SITT8 in tomato fruits might be responsible for the low accumulation of anthocyanins in WT and SlAN11 overexpression fruits, and modification of only one MBW member, like SIAN11, SITT8, SlANT1, and SlAN2, causes little effects in the fruit anthocyanin contents. Therefore, we speculated that coexpression of $M Y B$ and $b H L H$ genes may be an optimized transgenic strategy for improving anthocyanins accumulation in tomato fruits.

On the basis of our results and previous studies in model plant species, we proposed a hypothetical working model for SlAN11-dependent regulation of anthocyanin/PA accumulation and seed dormancy (Fig. 12). In tomato stems and leaves, SIAN11 interacts with bHLH TFs (SITT8 and SIGL3), and forms a MBW complex with MYB (SIANT1 and SIAN2) and bHLH TFs to regulate the transcription of SIDFR gene to control anthocyanin biosynthesis. In addition, for the absence of SIANT1, SIAN2, and SITT8 in tomato seeds, SlAN11 may interact with SlGL3 or other unknown bHLH TFs, and forms a MBW complex with unknown R2R3 Mybs and bHLH TFs to regulate the transcription of both SITTG2 and SIBAN genes to regulate PA biosynthesis in seed coats. Moreover, SlAN11 may participate in a complex with other proteins involved in seed dormancy to regulate the transcription of SlABI3 and SlABI5 genes to influence seed dormancy. However, the proteins that can interact with SIAN11 in tomato seeds are still unknown and require further investigation.

\section{Acknowledgements \\ The research was supported by the National Natural Science Foundation of China (No. 31500205, No. 31770644, and No.81703656), Scientific Research Fund of Sichuan Provincial Education Department (No. $17 Z$ B0456 and No.13TD0023), Yunnan Engineering Laboratory for Agro-environment Pollution Control and Eco-remediation/The Innovation Team for Farmland Non-pollution Production of Yunnan Province (No. 2017HC015), and the doctoral Fund Project (No. $14 z x 7157$ and No. 13zx7116) and Longshan academic talent research supporting program (No. 17LZXT09) of Southwest University of Science and Technology.}

\section{Author details}

${ }^{1}$ School of Life Science and Engineering, Southwest University of Science and Technology, Mianyang 621010, China. ${ }^{2}$ Yunnan Engineering Laboratory for Agro-environment Pollution Control and Eco-remediation, The Innovation Team for Farmland Non-pollution Production of Yunnan Province, Kunming 650201, China. ${ }^{3}$ Shaanxi Collaborative Innovation Center of Chinese Medicinal Resources Industrialization, Shaanxi University of Chinese Medicine, Xianyang 712046, China. ${ }^{4}$ Chengdu Institute of Biology, Chinese Academy of Sciences, Chengdu 610041, China

\section{Conflict of interest}

The authors declare that they have no conflict of interest.

Supplementary Information accompanies this paper at https://doi.org/ 10.1038/s41438-018-0032-3. 
Received: 28 November 2017 Revised: 3 March 2018 Accepted: 11 March 2018

\section{Published online: 01 June 2018}

\section{References}

1. Li, S. Transcriptional control of flavonoid biosynthesis: fine-tuning of the MYBbHLH-WD40 (MBW) complex. Plant Signal. Behav. 9, e27522 (2014).

2. Koes, R., Verweij, W. \& Quattrocchio, F. Flavonoids: a colorful model for the regulation and evolution of biochemical pathways. Trends Plant. Sci. 10, 236-242 (2005).

3. Ben-Simhon, Z. et al. A pomegranate (punica granatum I.) WD40-repeat gene is a functional homologue of Arabidopsis TTG1 and is involved in the regulation of anthocyanin biosynthesis during pomegranate fruit development. Planta 234, 865-881 (2011).

4. Nesi, N. et al. The TT8 gene encodes a basic helix-loop-helix domain protein required for expression of DFR and BAN genes in Arabidopsis siliques. Plant Cell 12, 1863-1878 (2000).

5. Gonzalez, A., Zhao, M., Leavitt, J. M. \& Lloyd, A. M. Regulation of the anthocyanin biosynthetic pathway by the TTG1/bHLH/Myb transcriptional complex in Arabidopsis seedlings. Plant J. 53, 814-827 (2008).

6. $\mathrm{Xu}$, W.et al. Complexity and robustness of the flavonoid transcriptional regulatory network revealed by comprehensive analyses of Myb-bHLH-WDR complexes and their targets in Arabidopsis seed. New Phytol. 202, 132-144 (2014).

7. Neer, E. J., Schmidt, C. J., Nambudripad, R. \& Smith, T. F. The ancient regulatory protein family of WD-repeat proteins. Nature 371, 297-300 (1994).

8. Stirnimann, C. U., Petsalaki, E., Russell, R. B. \& Müller, C. W. WD40 proteins propel cellular networks. Trends Biochem. Sci. 35, 565-574 (2010).

9. De Vetten, N., Quattrocchio, F., Mol, J. \& Koes, R. The an11 locus controlling flower pigmentation in petunia encodes a novel WD repeat protein conserved in yeast, plants, and animals. Genes Dev. 11, 1422-1434 (1997).

10. Walker, A. R. et al. The TRANSPARENT TESTA GLABRA1 locus, which regulates trichome differentiation and anthocyanin biosynthesis in Arabidopsis, encodes a WD40 repeat protein. Plant Cell 11, 1337-1349 (1999).

11. Ramsay, N. A. \& Glover, B. J. MYB-bHLH-WD40 protein complex and the evolution of cellular diversity. Trends Plant. Sci. 10, 63-70 (2005).

12. Carey, C. C., Strahle, J. T., Selinger, D. A. \& Chandler, V. L. Mutations in the pale aleurone color 1 regulatory gene of the Zea mays anthocyanin pathway have distinct phenotypes relative to the functionally similar TRANSPARENT TESTA GLABRA1 gene in Arabidopsis thaliana. Plant Cell 16, 450-464 (2004).

13. Pang, Y. et al. A WD40 repeat protein from Medicago truncatula is necessary for tissue-specific anthocyanin and proanthocyanidin biosynthesis but not for trichome development. Plant Physiol. 151, 1114-1129 (2009).

14. Schaart, J. G. et al. Identification and characterization of MYB-bHLH-WD40 regulatory complexes controlling proanthocyanidin biosynthesis in strawberry (Fragariaxananassa) fruits. New Phytol. 197, 454-467 (2013).

15. Chopra, D. et al. Analysis of TTG1 function in Arabisalpina. BMC Plant Biol. 14, 16 (2014).

16. Fiedor, J. \& Burda, K. Potential role of carotenoids as antioxidants in human health and disease. Nutrients 6, 466-488 (2014).

17. Jones, C. M., Mes, P. \& Myers, J. R. Characterization and inheritance of the anthocyanin fruit (Aft) tomato. J. Hered. 94, 449-456 (2003).

18. Mathews, $\mathrm{H}$. et al. Activation tagging in tomato identifies a transcriptional regulator of anthocyanin biosynthesis, modification, and transport. Plant Cell 15, 1689-1703 (2003).

19. Schreiber, G. et al. Anthocyanin 1 from Solanum chilense is more efficient in accumulating anthocyanin metabolites than its Solanum lycopersicum counterpart in association with the anthocyanin fruit phenotype of tomato. Theor. Appl. Genet. 124, 295-308 (2011).

20. Kiferle, C. et al. Tomato R2R3-MYB proteins SIANT1 and SIAN2: same protein activity, different roles. PLOS ONE 10, e0136365 (2015).

21. Nukumizu, Y., Wada, T. \& Tominaga-Wada, R. Tomato (Solanum lycopersicum) Homologs of TRIPTYCHON (SITRY) and GLABRA3 (SIGL3) are involved in anthocyanin accumulation. Plant Signal. Behav. 8, e24575 (2013).

22. Qiu, Z. et al. The tomato Hoffman's anthocyaninless gene encodes a bHLH transcription factor involved in anthocyanin biosynthesis that is developmentally regulated and induced by low temperatures. PLOS ONE 11, e0151067 (2016).

23. Farris, L. \& Edwards, R. in Molecular Cloning: A laboratory Manual3rd edn (eds Sambrook, J \& Russell, D. W.). New York: Cold Spring Harbor Laboratory Press, 2-137(2001).
24. Guo, H. S. et al. A chemical-regulated inducible RNAi system in plants. Plant J. Cell Mol. Biol. 34, 383-392 (2003).

25. Fillatti, J. A. J., Kiser, J., Rose, R. \& Comai, L. Efficient transfer of a glyphosate tolerance gene into tomato using a binary Agrobacterium tumefaciens vector. Biotechnology 5, 726-730 (1987).

26. Beeckman, T. \& Engler, G. An easy technique for clearing of histochemically stained plant tissue. Plant Mol. Biol. Report 82, 259-266 (1994).

27. Huang, W. et al. SINAC1, a stress-related transcription factor, is fine-tuned on both the transcriptional and the post-translational level. New Phytol. 197, 1214-1224 (2013).

28. Mustilli, A. C., Fenzi, F., Ciliento, R., Alfano, F. \& Bowler, C. Phenotype of the tomato high pigment- 2 mutant is caused by a mutation in the tomato homolog of DEETIOLATED1. Plant Cell 11, 145-157 (1999).

29. Mitsunaga, T., Doi, T., Kondo, Y. \& Abe, I. Color development of proanthocyanidins in vanillin-hydrochloric acid reaction. J. Wood Sci. 44, 125-130 (1998).

30. Sheahan, J. J. \& Rechnitz, G. A. Flavonoid-specific staining of Arabidopsis thaliana. Biotechniques 13, 880-883 (1992).

31. Gao, Y. et al. Functional characterization of two alternatively spliced transcripts of tomato ABSCISIC ACID INSENSITIVE3 (AB/3) gene. Plant Mol. Biol. 82, 131-145 (2013).

32. Ben-Simhon, Z. et al. A pomegranate (Punica granatum L.) WD40-repeat gene is a functional homologue of Arabidopsis TTG1 and is involved in the regulation of anthocyanin biosynthesis during pomegranate fruit development. Planta 234, 865-881 (2011).

33. An, X. H., Tian, Y., Chen, K. Q., Wang, X. F. \& Hao, Y. J. The apple WD40 protein MdTTG1 interacts with bHLH but not MYB proteins to regulate anthocyanin accumulation. J. Plant Physiol. 69, 710-717 (2012).

34. Zhao, M., Morohashi, K., Hatlestad, G., Grotewold, E. \& Lloyd, A. The TTG1bHLH-MYB complex controls trichome cell fate and patterning through direct targeting of regulatory loci. Development 135, 1991-1999 (2008).

35. Balkunde, R., Bouyer, D. \& Hülskamp, M. Nuclear trapping by GL3 controls intercellular transport and redistribution of $\mathrm{ttg} 1$ protein in Arabidopsis. Development 138, 5039-5048 (2011).

36. Shirley, B. W. et al. Analysis of Arabidopsis mutants deficient in flavonoid biosynthesis. Plant J. 8, 659-671 (1995).

37. Xie, D. Y. \& Dixon, R. A. Role of anthocyanidin reductase, encoded by BANYULS in plant flavonoid biosynthesis. Science 299, 396-399 (2003).

38. Nesi, N., Jond, C., Debeaujon, I., Caboche, M. \& Lepiniec, L. The Arabidopsis TT2 gene encodes an R2R3 MYB domain protein that acts as a key determinant for proanthocyanidin accumulation in developing seed. Plant Cell 13, 2099-2114 (2001).

39. Debeaujon, I. et al. Proanthocyanidin-accumulating cells in Arabidopsis Testa: regulation of differentiation and role in seed development. Plant Cell 15, 2514-2531 (2003).

40. Johnson, C. S., Kolevski, B. \& Smyth, D. R. TRANSPARENT TESTA GLABRA2, a trichome and seed coat development gene of Arabidopsis, encodes a WRKY transcription factor. Plant Cell 14, 1359-1375 (2002).

41. Finkelstein, R. R. \& Lynch, T. J. The Arabidopsis abscisic acid response gene ABI5 encodes a basic leucine zipper transcription factor. Plant Cell 12, 599-609 (2000).

42. Liu, X. et al. The MrWD40-1 gene of Chinese bayberry (Myricarubra) interacts with MYB and bHLH to enhance anthocyanin accumulation. Plant Mol. Biol. Report 31, 1-11 (2013).

43. Koornneef, M. The complex syndrome of ttg mutants. Arab. Inf. Serv. 18, 45-51 (1981).

44. Baudry, A., Caboche, M. \& Lepiniec, L. TT8 controls its own expression in a feedback regulation involving TTG1 and homologous MYB and bHLH factors, allowing a strong and cell-specific accumulation of flavonoids in Arabidopsis thaliana. Plant J. 46, 768-779 (2006).

45. Baudry, A. et al. TT2, TT8, and TTG1 synergistically specify the expression of banyuls and proanthocyanidin biosynthesis in Arabidopsis thaliana. Plant J. 39, 366-380 (2004)

46. Serna, L. \& Martin, C. Trichomes: different regulatory networks lead to convergent structures. Trends Plant. Sci. 11, 274-280 (2006).

47. Hülskamp, M. \& Kirik, V. Trichome differentiation and morphogenesis in Arabidopsis. Adv. Bot. Res. 31, 237-260 (2000).

48. Kang, J. H., Shi, F., Jones, A. D., Marks, M. D. \& Howe, G. A. Distortion of trichome morphology by the hairless mutation of tomato affects leaf surface chemistry. J. Exp. Bot. 61, 1053-1064 (2010). 
49. Debeaujon, I., Léonkloosterziel, K. M. \& Koornneef, M. Influence of the testa on seed dormancy, germination, and longevity in Arabidopsis. Plant Physiol. 122, 403-414 (2000).

50. Duran, J. M. \& Retamal, N. Coat structure and regulation of dormancy in Sinapis arvensis L. seeds. J. Plant Physiol. 135, 218-222 (1989).

51. Kantar, F., Pilbeam, C. J. \& Hebblethwaite, P. D. Effect of tannin content of faba bean (Vicia faba) seed on seed vigour, germination and field emergence. Ann. Appl. Biol. 128, 85-93 (1996).

52. Smýkal, P., Vernoud, V., Blair, M. W., Soukup, A. \& Thompson, R. D. The role of the testa during development and in establishment of dormancy of the legume seed. Front. Plant Sci. 5, e75678-e75678 (2014).

53. Kartzeva, T., Balacheva, E., Atanassova, B. \& Molle, E. Effect of three anthocyaninless genes on germination ability of the brown seed (bs) mutant in tomato. seed Sci. Technol. 40, 177-184 (2012).

54. Nambara, E. et al. A screen for genes that function in abscisic acid signaling in Arabidopsis thaliana. Genetics 161, 1247-1255 (2002).

55. McCarty, D. R., Carson, C. B., Stinar, P. S. \& Robertson, D. S. Molecular analysis of viviparous-1: an abscisic acid-insensitive mutant of maize. Plant Cell 1, 523-532 (1989).

56. Chen, $M$. et al. TRANSPARENT TESTA GLABRA1 regulates the accumulation of seed storage reserves in Arabidopsis. Plant. Physiol. 169, 391-402 (2015).
57. Jia, L. et al. Proanthocyanidins inhibit seed germination by maintaining a high level of abscisic acid in Arabidopsis thaliana. J. Integr. Plant Biol. 54, 663-673 (2012).

58. Dubos, C. et al. MYBL2 is a new regulator of flavonoid biosynthesis in Arabidopsis thaliana. Plant J. 55, 940-953 (2008).

59. Grotewold, E. et al. Identification of the residues in the MYB domain of maize C1 that specify the interaction with the bHLH cofactor R. Proc. Natl Acad. Sci. USA 97, 13579-13584 (2000).

60. Bernhardt, $C$. et al. The bHLH genes GLABRA3 (GL3) and ENHANCER OF GLABRA3 (EGL3) specify epidermal cell fate in the Arabidopsis root. Development 130, 6431-6439 (2003).

61. Zuluaga, D. et al. Arabidopsis thaliana MYB75/PAP1 transcription factor induces anthocyanin production in transgenic tomato plants. Funct. Plant Biol. $\mathbf{3 5}$ 606-618 (2008).

62. Meng, $X$. et al. Physiological changes in fruit ripening caused by overexpression of tomato SIAN2, an R2R3-MYB factor. Plant Physiol. Biochem. 89 24-30 (2015).

63. Ren, L. et al. Heterologous expression of BoPAP1 in tomato induces stamen specific anthocyanin accumulation and enhances tolerance to a long-term low temperature stress. J. Plant. Growth Regul. 33, 757-768 (2014). 